\title{
Integrated sleep scheduling for multiple relays and UEs in LTE-TDD
}

\author{
Chun-Chuan Yang ${ }^{1}$, Yi-Ting Mai $^{2}$, Jeng-Yueng Chen ${ }^{3^{*}}$ (I) and Chen-Ming Yang ${ }^{1}$
}

\begin{abstract}
Energy-saving for the LTE-A network with relay nodes in TDD mode is addressed in this paper, and integrated sleep scheduling schemes for relay nodes and user devices under a base station are designed. The authors' two previously proposed ideas, namely, Load-Based Power Saving (LBPS) and Virtual Time, are adopted in the design, and two strategies, namely, top-down and bottom-up each with three LBPS schemes are proposed. In the top-down strategy, the load as well as the channel quality on the backhaul link is first considered to determine the sleep pattern for all relay nodes, and then the sleep schedule for UEs under each relay node is determined accordingly. On the contrary, the load and the channel quality on the access links are first considered and then integrated into the sleep schedule on the backhaul link. Two associated mechanisms for the proposed LBPS schemes to operate in the virtual time domain are also proposed in the paper, i.e., calculation of the virtual subframe capacity and the mapping mechanisms from the virtual time to the actual time. The benefit of the proposed schemes in power saving over the standard-based scheme is demonstrated by the simulation study, and the bottom-up scheme of BU-Split outperforms the other schemes under equally distributed input load as well as the hotspot scenario. Discussion on the tradeoff of the processing overhead and the performance for the proposed schemes is presented in the paper.
\end{abstract}

Keywords: LTE-A, Relay node, TDD, Power saving

\section{Introduction}

\subsection{Motivation}

Long-Term Evolution [1], denoted by LTE, and its successor LTE-Advanced [2], denoted by LTE-A, have become the major mobile communications technology and have been rapidly deployed worldwide to provide versatile services and attract more users in recent years. In order to extend the coverage area and provide higher transmission rates for User Equipment (denoted by $U E$ ) at the cell edge, the idea of relay node (denoted by $R N$ ) was proposed in the standard of LTE-A. However, the introduction of RN in the LTE-A network inevitably complicates technical issues such as interference mitigation, radio resource management, and packet transmission scheduling.

On the other hand, since there are two transmission directions in LTE/LTE-A, namely, uplink (denoted by

\footnotetext{
* Correspondence: jychen@hust.edu.tw

${ }^{3}$ Department of Information Networking Technology, Hsiuping University of Science and Technology, Taichung City, Taiwan, Republic of China Full list of author information is available at the end of the article
}

$U L$ ) and downlink (denoted by $D L$ ), two modes of duplex transmission are specified in the standard of LTE/LTE-A: frequency division duplex (denoted by $F D D$ ) and time division duplex (denoted by TDD). In the mode of FDD, two different and sufficiently separated frequency bands are used for each of the directions respectively. In the mode of TDD, UL transmission and DL transmission share a single frequency band in a time-sharing manner. For the LTE network without RN, seven different TDD configurations are defined. Different numbers of DL and UL subframes are designated in different configurations in a time period of $10 \mathrm{~ms}$ to provide flexibility in resource management for both directions.

Combination of RN with TDD creates a complicated network environment since there are two radio links between DeNB and UEs. The radio link between DeNB and RNs is called the backhaul link. The radio link between an RN and its attached UEs is called the access link. Technical issues concerning about the combination of RN and TDD include (1) dynamic TDD configuration 
for the radio links, (2) radio resource allocation and assignment for the two-hop transmission, (3) interference reduction among the base stations, relays, and user devices, (4) energy-conserving mechanisms for user devices as well as the core network, etc.

\subsection{Methodology}

For many years, we have been researching the energysaving issue in mobile and wireless communications, and the idea of Load-Based Power Saving (denoted by $L B P S$ ) was proposed for sleep scheduling in the wireless network of IEEE 802.16 in our previous work [3]. The idea of LBPS was also extended to support sleep scheduling in LTE $[4,5]$. In this paper, we are focusing on a more general and complicated network environment in which there are a number of RNs under the same base station of DeNB in the mode of TDD. The challenge in designing a sleep scheduling mechanism for RNs and UEs in TDD lies in the two-hop transmission from the DeNB to UEs. There are two issues to be dealt with. Firstly, transmission scheduling and sleep scheduling for RNs and UEs should be properly coordinated in order to maximize power saving efficiency. Secondly, since the TDD configuration with the presence of $\mathrm{RN}$ affects not only the backhaul link but also the access link, there should be an efficient way to regulate the arrangement of resource on both links and to better support sleep scheduling. The first step in our methodology is to deal with TDD configuration by extending the authors' previously proposed idea of virtual time to support two-hop transmission. The second step is to design a hierarchical sleeping scheme based on LBPS to cover all RNs and attached UEs under the DeNB. As will be shown, there are two directions in the hierarchical design, top-down and bottom-up, each with three proposed sleep scheduling schemes. Power saving efficiency of the proposed schemes is demonstrated by the simulation study.

The rest of the paper is structured as in the following. Firstly, a brief introduction for LTE-A RN, TDD, and the previous work of LBPS is presented in Section 2. In Section 3, two strategies each with three integrated power saving schemes are explained. In Section 4, the results of performance comparison by simulation study are presented. The conclusion of the paper is presented in Section 5.

\section{Related work}

\subsection{LTE-A RN and TDD}

The advantages of using RN in LTE-A include increasing network density, extending network coverage, and rapid network roll-out. There are two methods of operation concerning the carrier frequency for an $\mathrm{RN}$ to operate on inband and outband. An inband RN uses the same frequency band for the backhaul link and the access link. The backhaul link and the access link of an outband RN operate on different carrier frequency bands. Two basic types of RN are proposed in LTE-A: Type 1 and Type 2. A Type $1 \mathrm{RN}$, which is a Layer 3 device, controls its cell with its own identity and transmits its own synchronization information as well as reference symbols. Type 1 RNs is inband RNs and provides half-duplex transmission for UL and DL directions. Moreover, a type $1 \mathrm{RN}$ acts as if it is a Release 8 eNodeB to the UEs for backward compatibility. A type $2 \mathrm{RN}$, which is a Layer 2 device, does not operate its own cell and acts as an extension of the main cell so that UEs cannot distinguish a type $2 \mathrm{RN}$ from the Donor eNB (DeNB). In this paper, we focus on type $1 \mathrm{RN}$.

When a type $1 \mathrm{RN}$ receives data from the DeNB on the backhaul link, Multimedia Broadcast Single-Frequency Network (MBSFN) subframes are used to mute transmission on the access link. That is, by designating some subframes on the access link as MBSFN subframes, a type $1 \mathrm{RN}$ transmits empty MBSFN subframes with only control signals to the UEs and then receives transmission from the DeNB. On the other hand, in order to provide flexibility for resource management of DL and UL transmissions, seven TDD configurations are defined in LTE for transmission between eNB and UEs as shown in Table 1. Different numbers and positions of DL and UL subframes are designated within a radio frame of $10 \mathrm{~ms}$ in different TDD configurations. Due to the requirements of MBSFN subframes and hybrid automatic repeat request (HARQ) operation, there are 19 configurations specified by 3GPP for type 1 RNs in the mode of TDD as displayed in Table 2 . Note that since subframes $0,1,5$, and 6 cannot be configured as MBSFN subframes, these four subframes cannot be used by the backhaul link, which excludes configuration 0 and configuration 5 to be used by the relay cell (i.e., the access link).

Research work involving RN and TDD in the literature includes (1) resource allocation schemes and packet transmission scheduling to achieve high system throughput and maintain fairness among UEs [6-8]. (2) Interference reduction mechanisms among neighboring base

\begin{tabular}{|c|c|c|c|c|c|c|c|c|c|c|}
\hline \multirow{2}{*}{$\begin{array}{l}\text { DL/UL } \\
\text { configuration }\end{array}$} & \multicolumn{10}{|c|}{ Subframe no. } \\
\hline & 0 & 1 & 2 & 3 & 4 & 5 & 6 & 7 & 8 & 9 \\
\hline 0 & $\mathrm{D}$ & S & $U$ & U & U & D & $S$ & U & U & U \\
\hline 1 & $\mathrm{D}$ & S & U & U & D & D & S & U & U & D \\
\hline 2 & $\mathrm{D}$ & S & U & D & D & $\mathrm{D}$ & $\mathrm{S}$ & U & $\mathrm{D}$ & D \\
\hline 3 & $\mathrm{D}$ & S & U & U & U & $\mathrm{D}$ & D & $\mathrm{D}$ & D & $\mathrm{D}$ \\
\hline 4 & $\mathrm{D}$ & S & U & U & D & $\mathrm{D}$ & D & $\mathrm{D}$ & $\mathrm{D}$ & D \\
\hline 5 & $\mathrm{D}$ & S & U & D & D & $\mathrm{D}$ & D & $\mathrm{D}$ & $\mathrm{D}$ & $\mathrm{D}$ \\
\hline 6 & $\mathrm{D}$ & S & $U$ & $U$ & U & $\mathrm{D}$ & $S$ & $U$ & U & $D$ \\
\hline
\end{tabular}

Note: $D$ DL subframe; U UL subframe; $S$ special subframe 
Table 2 Backhaul and access link TDD configuration in LTE-A

\begin{tabular}{|c|c|c|c|c|c|c|c|c|c|c|c|}
\hline \multirow{2}{*}{$\begin{array}{l}\text { Backhaul link } \\
\text { configuration }\end{array}$} & \multirow{2}{*}{$\begin{array}{l}\text { Access link } \\
\text { configuration }\end{array}$} & \multicolumn{10}{|c|}{ Subframe no. } \\
\hline & & 0 & 1 & 2 & 3 & 4 & 5 & 6 & 7 & 8 & 9 \\
\hline 0 & 1 & & & & & D & & & & U & \\
\hline 1 & & & & & $U$ & & & & & & $\mathrm{D}$ \\
\hline 2 & & & & & & D & & & & U & $\mathrm{D}$ \\
\hline 3 & & & & & $U$ & D & & & & & D \\
\hline 4 & & & & & $U$ & D & & & & U & $\mathrm{D}$ \\
\hline 5 & 2 & & & $U$ & & & & & & D & \\
\hline 6 & & & & & $D$ & & & & U & & \\
\hline 7 & & & & $U$ & & D & & & & D & \\
\hline 8 & & & & & $\mathrm{D}$ & & & & U & & D \\
\hline 9 & & & & $U$ & $D$ & D & & & & D & \\
\hline 10 & & & & & $\mathrm{D}$ & & & & U & D & $\mathrm{D}$ \\
\hline 11 & 3 & & & & $U$ & & & & $D$ & & $\mathrm{D}$ \\
\hline 12 & & & & & $U$ & & & & D & D & $\mathrm{D}$ \\
\hline 13 & 4 & & & & U & & & & & & D \\
\hline 14 & & & & & $U$ & & & & $\mathrm{D}$ & & $\mathrm{D}$ \\
\hline 15 & & & & & U & & & & & D & D \\
\hline 16 & & & & & U & & & & D & D & D \\
\hline 17 & & & & & $U$ & $\mathrm{D}$ & & & $\mathrm{D}$ & D & $\mathrm{D}$ \\
\hline 18 & 6 & & & & & U & & & & & D \\
\hline
\end{tabular}

stations and relays [9-12]. (3) Dynamic TDD configuration mechanisms adapting to different traffic loads in heterogeneous wireless networks [13-15]. (4) Mechanisms of resource management and allocation for coexisting FDD and TDD systems [16, 17].

\subsection{Previous work of LBPS}

In LBPS (load-based power saving), traffic flows are assumed to be Poisson for the benefit of multiplexing property in traffic modelling. As illustrated in Fig. 1, the load of the downlink traffic for $\mathrm{UE}_{1}$ is estimated and is used to calculate the length of the sleep period. Two threshold parameters are defined in LBPS: Data TH and Prob_TH. LBPS calculates the length of the sleep period for $\mathrm{UE}_{1}$ (i.e., $K$ time units as displayed in Fig. 1) such that the amount of data accumulation exceeds Data_TH with a probability higher than Prob_ $\mathrm{TH}$. In order to maintain a balance between power-saving efficiency and delay performance, the value of Data_TH is suggested to be the amount of data that can be transmitted in an LTE subframe. Moreover, Channel Quality Indicator (CQI) was used for estimating the capacity of a subframe $[4,5]$.

Three LBPS schemes, that is, LBPS-Aggr, LBPS-Split, and LBPS-Merge, were designed to multiplex multiple UEs in sleep scheduling. As the most basic scheme,
LBSP-Aggr aggregates the traffic flows of all UEs in determining the length of the sleep period and all UEs are synchronized in the sleep pattern. The enhanced scheme of LBPS-Split tries to split the UEs into different groups in sleep scheduling in order to lengthen the sleep period and increase power-saving efficiency. On the other hand, LBPS-Merge initially treats each UE as a single-member group and merges some of the groups until a feasible sleep schedule is found. It's worth mentioning that the length of the sleep period for each group in LBSP-Merge is set as a power of 2 (subframes) for an efficient check on the schedulability in sleep scheduling. For details of LBPS schemes, please refer to our previous work $[3,4]$.

The three LBPS schemes mentioned above were originally designed for LTE-FDD with the assumption that every subframe is available in a continuous manner. However, the assumption is no longer valid in the mode of TDD since the availability of subframes for DL and UL transmission depends on the TDD configuration. Therefore, in order for the LBPS schemes to be effectively applied to LTE-TDD, the idea of Virtual Time was proposed by the authors [5]. In the domain of virtual time, the subframes (virtual subframes) are available in a continuous manner so that the LBPS schemes can operate without any modification. That is, for LTE-TDD, the sleep schedule in the domain of virtual time is first determined by LBPS schemes and then mapped to the actual time. Three mapping mechanisms were investigated in the authors' previous work: 1-to-all mapping, continuous mapping, and 1-to-1 first mapping. We have found that 1-to-1 first mapping performs better than the other two methods in power saving.

\section{Proposed integrated sleep scheduling}

\subsection{Basic idea}

As shown in Fig. 2, we focus on the network environment in which multiple RNs (denoted by $\mathrm{RN}_{1} \sim \mathrm{RN}_{\mathrm{n}}$, and $n$ is the number of RNs) are under the same DeNB, and a number of UEs are associated with each RN. The TDD configuration on the backhaul link is denoted by Config- $B$. The TDD configuration for each of the access link (denoted by $A_{1} \sim A_{n}$ ) is denoted by Config- $A_{i}$. The idea of Virtual Time from the authors' previous work is adopted in this paper in order for the proposed schemes to be applied easily for the combination of $\mathrm{RN}$ and TDD. Based on the calculation of virtual subframe capacity (denoted by VSC) for both the backhaul link and the access links, two strategies for designing the integrated sleep schedule, namely, top-down and bottomup, are considered.

In the top-down strategy, the load as well as the channel quality on the backhaul link are first considered to determine the sleep pattern for all RNs, and 


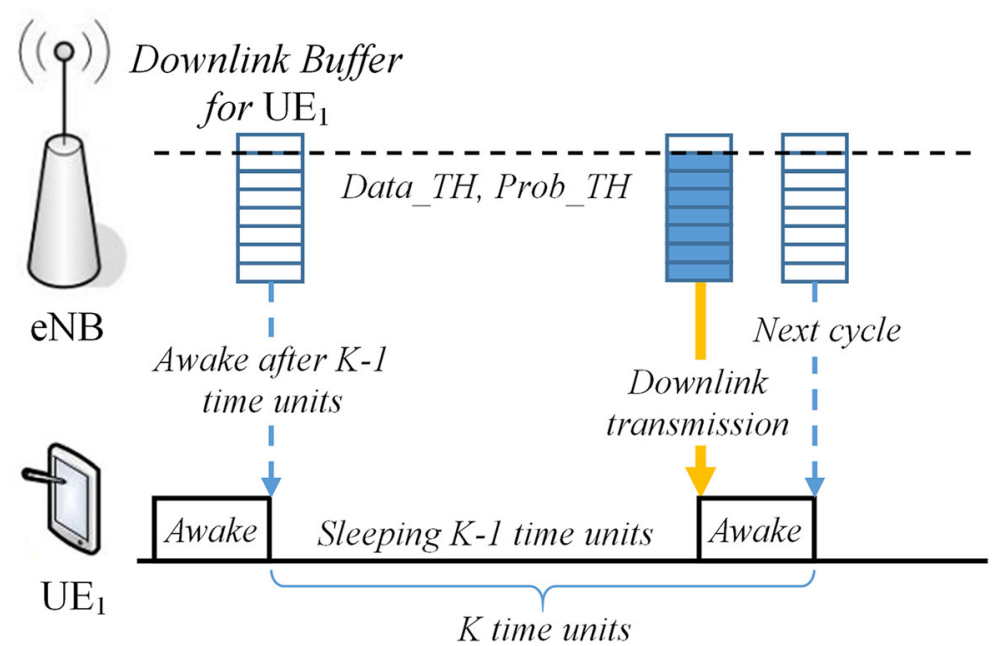

Fig. 1 Load-based power saving — the load of the downlink traffic for UE1 which is estimated and is used to calculate the length of the sleep period

then the sleep pattern for UEs under each RN is decided accordingly. On the contrary, the load and the channel quality on the access links are first considered and then integrated to the sleep schedule on the backhaul link. Three top-down schemes, denoted by TD-Aggr, TD-Split, and TD-Merge, and three bottomup schemes, denoted by $B U$-Aggr, BU-Split, and $B U$ Merge, are proposed in this paper. In the following sections, VSC calculation, top-down schemes, bottomup schemes, and the mapping mechanisms are presented respectively.

\subsection{Calculation of virtual subframe capacity}

The operation of the proposed LBPS schemes in the domain of virtual time is based on the calculation of virtual subframe capacity, which is used in setting the value of Data_TH as well as in estimating the length of the sleep period. The radio resource in a virtual subframe comes from the available radio resource according to the TDD configuration. For instance, as displayed in Fig. 3, the total amount of DL radio resource on the backhaul link for Configuration 17 is four DL subframes, i.e., subframe $4,7,8$, and 9. The total radio resource of the four subframes is equally allocated to each virtual subframe.

Moreover, the resource sharing constraint of type 1 $\mathrm{RN}$ indicates that a DL subframe cannot be used by the backhaul link and the access link at the same time, and we assume that the backhaul link has a higher priority to use the DL radio resource than the access links. Hence, the DL resource that can be used Fig. 2 Proposed integrated sleeping scheduling for RNs and UEs-the idea of virtual time is adopted in this paper in order for the proposed 
E.g. Configuration 17 for the backhaul link (DL only)
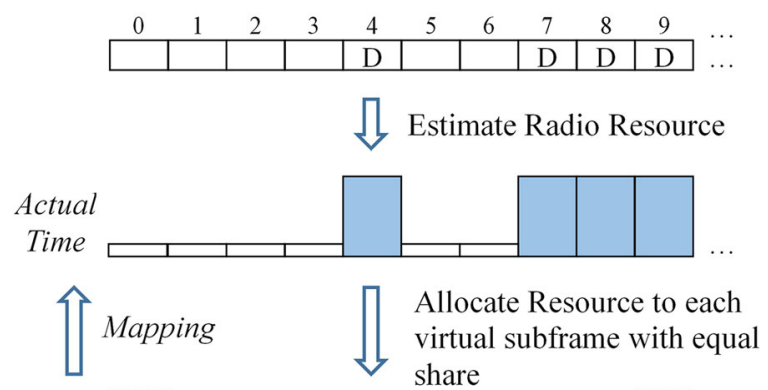

Virtual Time

Fig. 3 The idea of virtual time--the operation of the proposed LBPS schemes in the domain of virtual time is based on the calculation of virtual subframe capacity, which is used in setting the value of Data_TH as well as in estimating the length of the sleep period

for transmission on the access link should exclude the same resource that are assigned on the backhaul link. That is, the average number of subframe on an access link is calculated as the total number of DL subframe in the TDD configuration minus the expected number of the same subframes used on the backhaul link. VSC for the backhaul link (denoted by $\mathrm{VSC}_{\mathrm{Bk}}$ ) and VSC for the access links (denoted by $\mathrm{VSC}_{\mathrm{Ai}}$ ) is calculated according to the average number of subframe that can be used on the link, as presented in the following.

$$
\begin{aligned}
\mathrm{VSC}_{\mathrm{Bk}}= & \frac{1}{10}(\text { Avg. subframe capacity of } \mathrm{Bk}) \\
& \times(\text { \#DL subframes in Config-B }) \\
\mathrm{VSC}_{\mathrm{Ai}}= & \frac{1}{10}\left(\text { Avg. subrame capacity of } \mathrm{A}_{i}\right) \\
& \times\left(\text { Avg.\#DL subframe in } \mathrm{A}_{i}\right)
\end{aligned}
$$

(Avg. \#DL subframes in $\left.\mathrm{A}_{i}\right)=\left(\# \mathrm{DL}\right.$ subframes in Config- $\left.\mathrm{A}_{i}\right)$ -(\#Same DL subframes used by $\mathrm{Bk}) \times($ Backhaul Utilization $)$

$$
(\text { Backhaul Utilization })=\frac{\text { Total DL Input Load }}{\text { Total DL Capacity of Bk }}
$$

For instance, given Configuration 17 for the backhaul link (and therefore Configuration 4 for each access link $A_{i}$ ), "\#DL subframes in Config- $B$ " is 4 and "\#DL subframes in Config- $A_{i}$ " is 8 . In this paper, the special subframe is seen as a DL subframe for simplicity, and therefore, "\#Same DL subframes used by BK" is 4. The average subframe capacity (Avg. Subframe Capacity of $B k$ and $A_{i}$ ) is calculated according to the total system bandwidth, the bandwidth used by the control channels, and the link quality [4]. In the following, proposed top-down and bottom-up schemes are presented and the notations used are summarized in Table 3. Note that we assume the RNs are appropriately positioned so that there is no interference among the relay cells, and therefore, the sleep schedule on each access link can be decided independently.

\subsection{Top-down LBPS schemes}

The first step in the top-down schemes is determining the sleep schedule of all RNs on the backhaul link according to the load and $\mathrm{VSC}_{B k}$. The second step is determining the sleep schedule of each $\mathrm{RN}$ and the attached UEs on the access link. Three top-down LBPS schemes of TD-Aggr, TD-Split, and TD-Merge are designed and explained as follows.

In the first step, the sleep cycle length for each RN on the backhaul link is calculated according to $\mathrm{VSC}_{\mathrm{Bk}}$ and each RN's DL load $\left(\lambda_{i}\right)$. All RNs are treated as a group in the sleep schedule in TD-Aggr, and a number of groups is made in TD-Split and TD-Merge to increase the sleep cycle length. The threshold of data accumulation is set as virtual subframe capacity on the backhaul link, i.e., Data_TH $=\mathrm{VSC}_{\mathrm{Bk}}$. Figure 4 illustrates the examples of the first step in the three top-down schemes. The sleep patterns obtained in the first step merely indicates the behavior of RNs on the backhaul link that is integrated with the behavior of $\mathrm{RN}$ on the access link in the second step in order to decide the final sleep pattern.

Based on the result from the first step, the second step for the three top-down schemes is similar. Each RN needs to estimate the number of subframes required for transmitting the accumulated DL data to its attached UEs within one sleep cycle. The estimated number of subframes $N_{\mathrm{Ai}}$ on access link $A_{i}$ is calculated by dividing the amount of accumulated data at $\mathrm{RN}_{i}$ by the virtual subframe capacity of $A_{i}\left(V S C_{A i}\right)$. Since in the first step, the amount of accumulated data for a sleep group in

\begin{tabular}{|c|c|}
\hline Notation & Definition(unit) \\
\hline$V_{S C} C_{B k}$ & Backhaul subframe capacity (bit per subframe) \\
\hline $\mathrm{VSC}_{\mathrm{Ai}}$ & $\begin{array}{l}\text { Subframe capacity for access link } A_{i} \text { of } \mathrm{RN}_{i} \\
\text { (bit per subframe) }\end{array}$ \\
\hline$\lambda_{i}$ & $\mathrm{DL}$ arrival rate for $R N_{i}$ (bit per subframe) \\
\hline$N_{\mathrm{Ai}}$ & $\begin{array}{l}\text { Estimated number of virtual subframe for } \mathrm{RN}_{\mathrm{i}} \\
\text { to transmit accumulated DL data to UEs in a sleep cycle }\end{array}$ \\
\hline$N_{\mathrm{Bk}-\mathrm{Ai}}$ & $\begin{array}{l}\text { Estimated number of virtual subframe for DeNB } \\
\text { to transmit the accumulated } \mathrm{DL} \text { data to } \mathrm{RN}_{\mathrm{i}} \text { on } \\
\text { the backhaul link in a sleep cycle }\end{array}$ \\
\hline DataAcc $_{i}$ & $\begin{array}{l}\text { Estimated amount of accumulated } \mathrm{DL} \text { data for } \mathrm{RN}_{\mathrm{i}} \\
\text { in a given cycle time (bit) }\end{array}$ \\
\hline
\end{tabular}
one cycle is the capacity of one virtual subframe on the backhaul link $\left(\mathrm{VSC}_{\mathrm{Bk}}\right)$, the amount of accumulated data at each $\mathrm{RN}_{\mathrm{i}}$ in one sleep cycle is calculated by weighting

Table 3 Notations used in the proposed schemes 


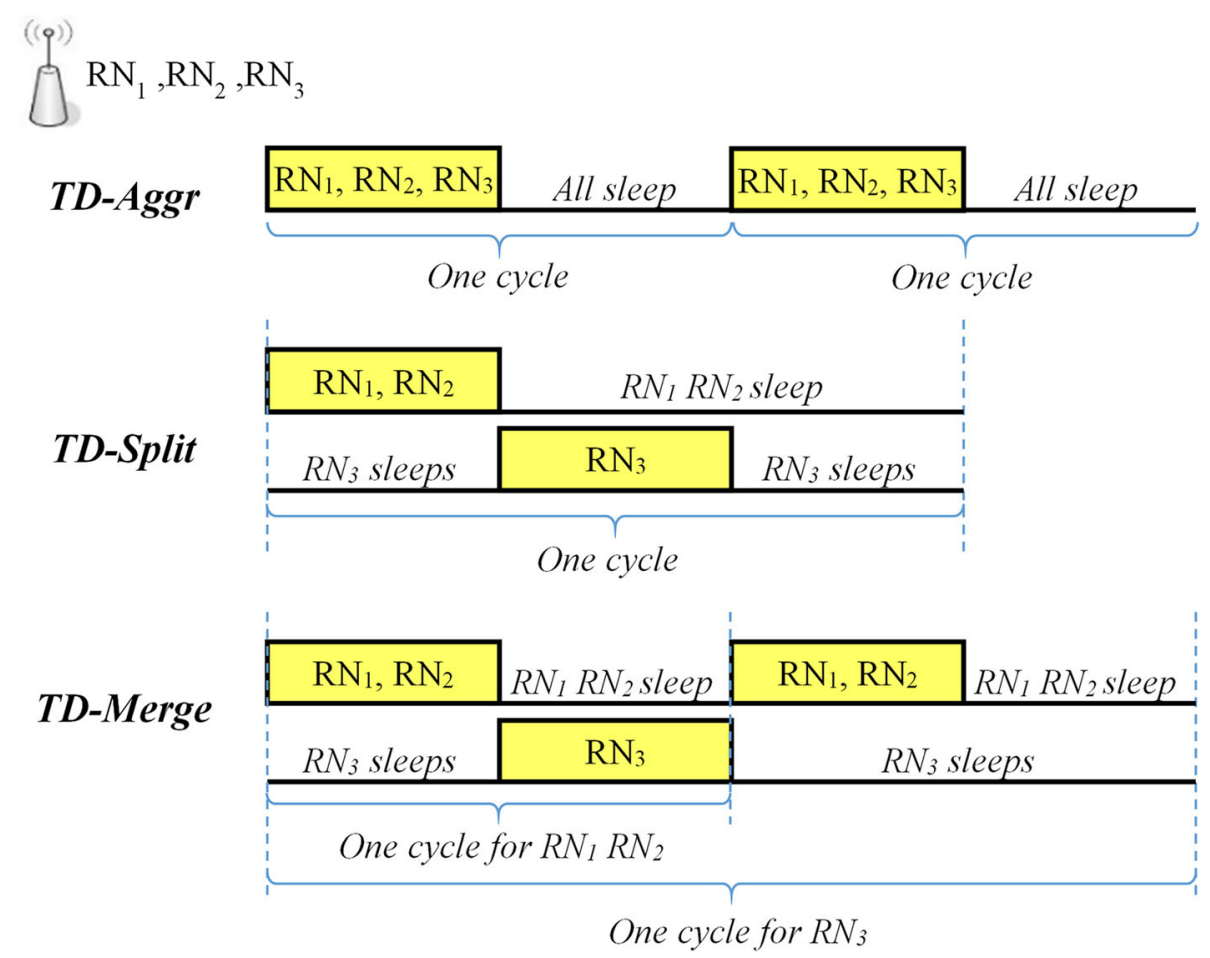

Fig. 4 First step in the top-down schemes-the first step in the top-down schemes is determining the sleep schedule of all RNs on the backhaul link

the load of $\mathrm{RN}_{\mathrm{i}}\left(\lambda_{i}\right)$ over the total load of all RNs in the same group. Calculation of $N_{A i}$ is presented in the following equation.

$$
N_{A i}=\left\lceil\frac{\left(\frac{\lambda_{i}}{\left.\sum_{\forall R N_{j} \text { in the same group of } R N_{i}{ }^{\lambda} j}\right) \times V S C_{B k}}\right.}{V S C_{A i}}\right\rceil
$$

Finally, schedulability check for each access link is performed by the following condition: if $\left(1+N_{\mathrm{Ai}}\right) \leq$ the cycle length of $R N_{i}$, a workable sleep schedule is found for $A_{i}$, meaning that the cycle length of $\mathrm{RN}_{\mathrm{i}}$ is big enough for it to receive data from DeNB (with 1 virtual subframe) and transmit data to UEs (with $N_{\mathrm{Ai}}$ virtual subframes). For example, given $\mathrm{N}_{\mathrm{Ai}}=2$ in Fig. 5 , the UEs under $\mathrm{RN}_{\mathrm{i}}$ are separated into two groups in the sleep schedule, and there are 3 virtual subframes in total for $\mathrm{RN}_{\mathrm{i}}$ to be awake for operation. If any of the access links fails to pass the schedulability check, the LBPS scheme ends with no power saving for all RNs and UEs in the virtual time domain.

\subsection{Bottom-up LBPS schemes}

The first step in the bottom-up schemes is to collect the sleep pattern suggested by each access link. Since different access link may have a different sleep pattern, the second step is to find a proper sleep cycle that can accommodate all RNs, which may lead to the adjustment of the sleep pattern on some access links. Therefore, the bottom-up schemes are more complicated than the topdown schemes. Three bottom-up schemes, namely, BUAggr, BU-Split, and BU-Merge, are proposed as explained in the following.

In BU-Aggr, the first step is to calculate the sleep cycle length for each access link based on the original LBPS-Aggr algorithm. The second step is to set the common sleep cycle length $K^{*}$ as the minimal cycle length among the access links and re-estimate the accumulated amount of data for each access link and check the schedulability for each RN and the schedulability for DeNB on the backhaul link. For each RN, it is schedulable if the cycle length $K^{\prime \prime}$ is larger than the total number of subframes required for the $\mathrm{RN}$ to receive data from DeNB and to transmit the data to UEs. For DeNB, it is schedulable if the cycle length $K^{*}$ is larger than the total number of subframes required to transmit the accumulated data to all RNs in a cycle. If any of the schedulability check fails, BUAggr fails to find a feasible integrated sleep schedule for all RNs and UEs. The algorithm of BU-Aggr is displayed in Fig. 6.

The original algorithm of LBPS-Split splits the UEs into as many groups as possible in order to increase power-saving efficiency. However, in the first step of 


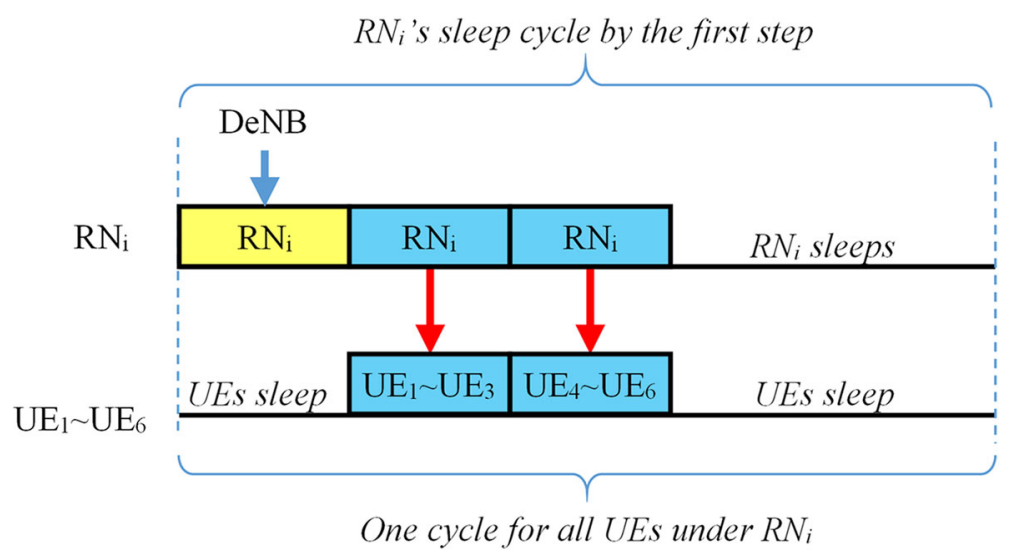

Fig. 5 Second step in the top-down schemes-the second step for the three top-down schemes is similar. Each RN needs to estimate the number of subframes required for transmitting the accumulated $D L$ data to its attached UEs within one sleep cycle

BU-Split, the number of UE groups under each RN is limited by the constraint that the cycle length must be large enough for the $\mathrm{RN}$ to receive accumulated data from DeNB as well as transmit the data to all UE groups in a cycle. In the second step of BU-Split, the final cycle length for all RNs is set as the minimal cycle length among RNs from the first step, and the group number of UEs under each RN is adjusted accordingly. As in BU-Aggr, the schedulability check for each RN and the schedulability check for DeNB on the backhaul link must be successful in order to have a feasible integrated sleep schedule for all RNs and UEs. The algorithm of BU-Split is displayed in Fig. 7, in which the Step 2 of BU-Split is the same as the Step 2 of BU-Aggr.

The basic idea of LBPS-Merge is to allow different cycle lengths for different groups in the sleep schedule, and the cycle length must be a power of 2 for efficient schedulability check. Based on the algorithm of LBPS-Merge, the first step in the proposed scheme of BU-Merge is to determine the cycle length of the UE groups for each access link. For each RN and its access link, schedulability check is performed in each iteration of the merge process to make sure a feasible sleep schedule can be found to accommodate the subframes required for the $\mathrm{RN}$ to receive data from DeNB and the subframes required for the RN to transmit data to the UE groups. When the first step is finished, the cycle length of each $\mathrm{RN}$ is determined, which may be different from the cycle length of other RNs. In the second step, the final cycle length is set as the maximal cycle length among RNs, and the schedulability on the backhaul link must be passed in order to have enough subframes for DeNB to transmit the accumulated data to all RNs in a cycle. The algorithm of BU-Merge is displayed in Fig. 8.

Step 1. For each access link $A_{i}$, calculate the initial sleep cycle length $\boldsymbol{K}_{i}$ by LBPS-Aggr with $\boldsymbol{D a t a}_{\boldsymbol{B}} \boldsymbol{T H} \boldsymbol{H}_{\boldsymbol{i}}=\boldsymbol{V} \boldsymbol{S} \boldsymbol{C}_{\boldsymbol{A} i}$

Step 2. The final cycle length: $\boldsymbol{K}^{*}=\operatorname{Min}\left\{\boldsymbol{K}_{i}\right\}$

For each $R N_{i}$, estimate the accumulated amount of data in a cycle: DataAcci $_{i}$

The number of subframe needed for DeNB to transmit data to $R N_{i}$ in a cycle: $\boldsymbol{N}_{B \boldsymbol{B}-\boldsymbol{A} i}=\left\lceil\frac{\text { DataAcc }_{i}}{V S C_{B k}}\right\rceil$

The number of subframe needed for $R N_{i}$ to transmit data to the UEs in a cycle: $\boldsymbol{N}_{A i}=\left\lceil\frac{\operatorname{DataAc}_{i}}{V S C_{A i}}\right\rceil$

Schedulability check for $R N_{i}: \boldsymbol{N}_{\boldsymbol{B} \boldsymbol{k}-\boldsymbol{A} \boldsymbol{i}}+\boldsymbol{N}_{\boldsymbol{A} i} \leq \boldsymbol{K}^{*}, \forall i$ (check if $R N_{i}$ can receive the accumulated data from DeNB and transmit the data to its UEs in a cycle)

Schedulability check for DeNB: $\left\lceil\frac{\sum_{i} D a t a A c c_{i}}{V S C_{B k}}\right\rceil \leq \boldsymbol{K}^{*}$ (check if DeNB can transmit the accumulated data to all RNs in a cycle)

If any of the above schedulability checks fails, the algorithm fails to find a feasible sleep schedule

Fig. 6 Algorithm of BU-Aggr-the algorithm detail for BU-Aggr scheme 
Step 1. For each access link $A_{i}$, calculate the initial sleep cycle length $\boldsymbol{K}_{\boldsymbol{i}}$ and the group number $\boldsymbol{M}_{\boldsymbol{i}}$ by LBPS-Split with

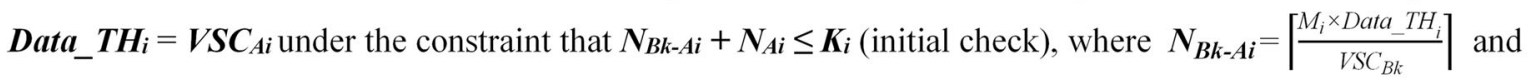
$N_{A i}=\left\lceil\frac{M_{i} \times D_{\text {Data }} T H_{i}}{V S C_{A i}}\right\rceil$

Step 2. (It's the same as the Step 2 of BU-Aggr)

Fig. 7 Algorithm of BU-Split—-the algorithm detail for BU-Split scheme

\subsection{Mapping mechanism}

Mapping from a virtual subframe to the actual subframe(s) depends on how the available radio resource in a TDD configuration is allocated to the virtual subframe. In the authors' previous work [5], two methods of resource allocation were investigated, namely, continuous mapping and 1-to-1 first mapping. In continuous mapping, the available radio resource is allocated to each virtual subframe with an equal share in a continuous manner. An example of continuous mapping for TDD configuration 4 (C4) is given in Fig. 9a. 1-to-1 first mapping goes for 1-to-1 mapping first and combine the remainder resource for allocation, as shown in Fig. 9b. Simulation results in our previous work showed that 1-to-1 first mapping performs better than continuous mapping in power saving for the LTE network without RNs.
With the presence of type $1 \mathrm{RNs}$ under the DeNB, there is one more issue to be dealt with. There are two different jobs for the $\mathrm{RN}$ in the two-hop transmission of DL: receiving data from the DeNB on the backhaul link and transmitting data to UEs on the access link. The two jobs of RN are scheduled in different virtual subframes by the proposed LBPS schemes, but as illustrated in Fig. 10, different virtual subframes could possibly be mapped to the same actual subframe and therefore only one of the two jobs can be done by the $\mathrm{RN}$. We call the problem $R N$ collision. The number of RN collisions should be reduced since it lowers RN's performance and may result in unexpected consequence. As presented in Section 4 of performance evaluation, 1-to-1 first mapping outperforms continuous mapping in terms of fewer $\mathrm{RN}$ collisions and achieves higher power-saving efficiency.

Step 1. For each access link $A_{i}$, starting from each UE as a group and setting $\boldsymbol{D a t a} \_\boldsymbol{T H} \boldsymbol{i}=\boldsymbol{V} \boldsymbol{S C}_{\boldsymbol{A} \boldsymbol{i}}$ for each group, calculate the sleep cycle length $\boldsymbol{K}_{G j}$ (a power of 2) for each group $\boldsymbol{G}_{j}$.

For each iteration in the merge process: (based on the idea of LBPS-Merge)

$$
\boldsymbol{K}_{i}=\operatorname{Max}\left\{\boldsymbol{K}_{G j}\right\}
$$

The number of subframe needed for DeNB to transmit data to $R N_{i}$ in $\boldsymbol{K}_{i}: \boldsymbol{N}_{\boldsymbol{B k}-A i}=\left|\frac{\text { Data }_{-} T H_{i} \times \sum_{G j} \frac{K_{i}}{V_{K_{G j}}}}{V S C_{B k}}\right|$

$$
\text { Schedulability check for } R N_{i}: \frac{N_{B k-A i}}{K_{i}}+\sum_{G j} \frac{1}{K_{G j}} \leq 1
$$

If the schedulability check is passed, go to Step 2.

If the schedulability check fails, a new merge operation is performed until the schedulability check is passed, or declare the algorithm fails when reaching the boundary condition of merge operation.

Step 2. The final cycle length: $\boldsymbol{K}^{*}=\operatorname{Max}\left\{\boldsymbol{K}_{i}\right\}$

For each $R N_{i}$ with a number of group $G_{j}$ (cycle length $K_{G j}$ ),

$$
\text { the accumulated data in a cycle of } \boldsymbol{K}^{*} \text { subframes: } \text { DataAcc }_{\boldsymbol{i}}=\text { Data_TH } H_{i} \times \sum_{G j} \frac{K^{*}}{K_{G j}}
$$

Schedulability check for DeNB: $\left[\frac{\sum_{i} D a t a A c c_{i}}{V S C_{B k}}\right] \leq \boldsymbol{K}^{*}$ (check if DeNB can transmit the accumulated data to all

$$
\text { RNs in a cycle of } \boldsymbol{K}^{*} \text { subframes) }
$$

If the schedulability check fails, the algorithm fails to find a feasible sleep schedule

Fig. 8 Algorithm of BU-Merge - the algorithm detail for BU-Merge scheme 


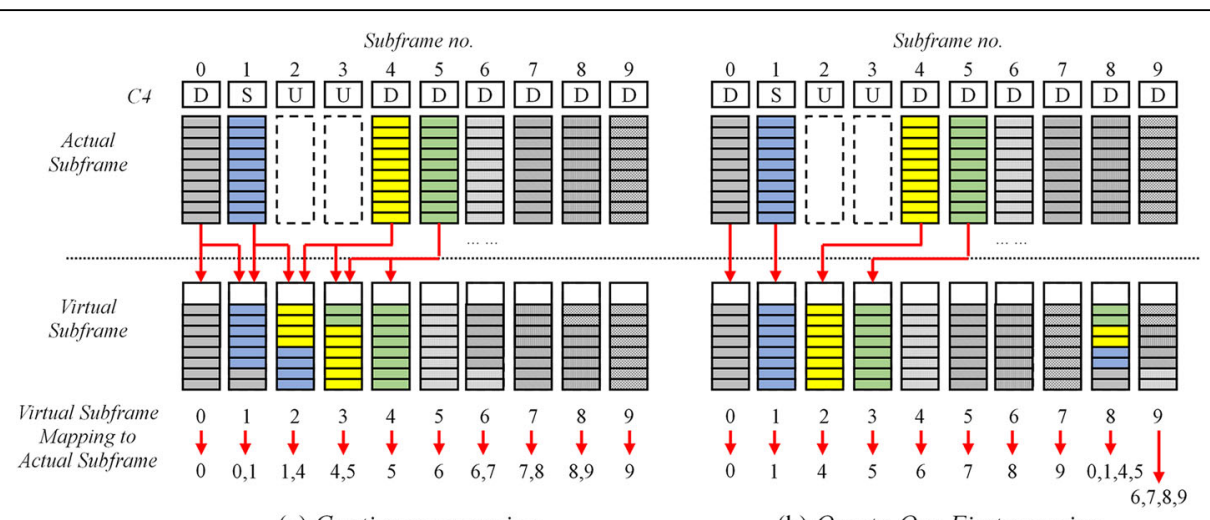

(a) Continuous mapping

(b) One-to-One First mapping

Fig. 9 Continuous mapping vs. 1-to-1 first mapping for C4-mapping example for Table 1

\section{Simulation results and discussion}

Simulation study was used to compare the performance of the proposed schemes. There are 6 RNs and 240 UEs in total under the DeNB. Two types of load distribution are investigated: equal load and hot spot. In equal load, there is the same number of UEs under each RN, i.e., 40 UEs. In hot spot, $80 \%$ of UEs is attached to 2 RNs, and $20 \%$ of UEs is attached to the other 4 RNs, i.e., two RNs are each with 96 UEs and four RNs are each with 12 UEs. A standard-based scheme, denoted by Std-DRX, is also included in the simulation. Simulation parameters are listed in Table 4.

\subsection{Comparison of the mapping mechanisms}

In order to compare the two mapping mechanisms, continuous mapping and 1-to-1 first mapping, TD-Aggr is selected as the sleep scheduling scheme and the values of CQI for the backhaul link and the access links are fixed. Two performance criteria are defined for comparison: $R N$ collision ratio and power-saving efficiency (denoted by PSE).
RN collision ratio is defined as the ratio of RN's awake subframes with the problem of RN collision as explained in Section 3.5. PSE is defined as the ratio of time for RN/UE in the sleep mode. Figures 11 and 12 show the results of $\mathrm{RN}$ collision ratio and PSE for TD-Aggr under the case of backhaul link CQI = 15 and access link $C Q I=13$. Another set of results under the case of backhaul link CQI = 10 and access link CQI = 9 is displayed in Figs. 13 and 14. The figures show that 1-to-1 first mapping can achieve lower RN collision ratio and higher PSE than continuous mapping. We conclude that 1-to-1 first mapping is a better mapping mechanism and is adopted for the following simulations.

\subsection{Results under equal load}

In this section, we compare the performance of the proposed schemes under equal load. The backhaul link CQI is randomly selected from 10 to 15 , and the access link CQI is randomly selected from 7 to 9 in the simulation. PSE of RN and UE of the proposed top-down schemes as well as the contrast scheme Std-DRX is displayed in Fig. 15. The average delay of downlink transmission for

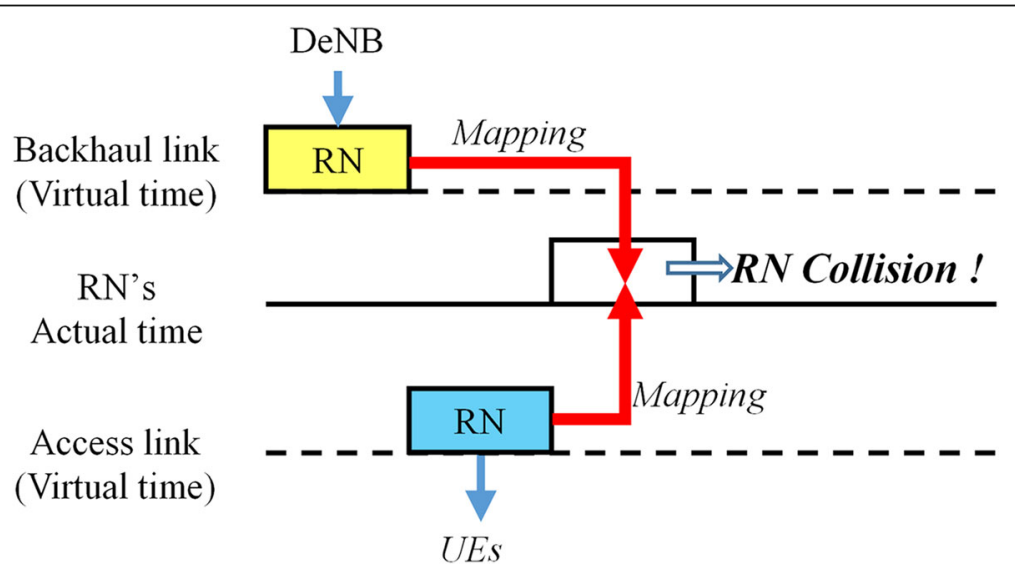

Fig. 10 The problem of RN collision—different virtual subframes could possibly be mapped to the same actual subframe and therefore only one of the two jobs can be done by the RN. We call the problem RN collision 
Table 4 Simulation parameters

\begin{tabular}{ll}
\hline System bandwidth & $20 \mathrm{MHz}(\# \mathrm{RB}=100)$ \\
\hline (\#RN, \#UE) & $(6,240)$ \\
Load distribution & Equal load, hot spot \\
Backhaul link quality & $\mathrm{CQI} 10 \sim 15$ \\
Access link quality & CQI $7 \sim 9$ \\
TDD configuration & $\mathrm{C} 17$ (C4 for access links) \\
Packet size & 800 bits
\end{tabular}

LBPS schemes parameters

Data_TH

Threshold of data accumulation

Prob_TH

0.8

Contrast scheme Std-DRX parameters

On duration $=1 \mathrm{~ms}$, inactivity timer $=40 \mathrm{~ms}$,

short DRX cycle $=80 \mathrm{~ms}$, short cycle timer $=1$, long DRX cycle $=160 \mathrm{~ms}$

the top-down schemes is displayed in Fig. 16. PSE and the average delay of the bottom-up schemes are displayed in Figs. 17 and 18. Some observations can be made from the figures:

(1) Proposed schemes are better than the contrast scheme of Std-DRX in terms of RN's PSE as well as UE's PSE, and in terms of the average delay, which shows the benefit of regulated sleep scheduling.

(2) Among top-down schemes, TD-Split and TDMerge outperform TD-Aggr in terms of RN's and
UE's PSE, which shows the benefit of grouping in sleep scheduling. However, as displayed in Fig. 16, higher PSE results in higher average delays. Similarly, BU-Split and BU-Merge achieve higher PSE than BU-Aggr at the cost of higher delays.

(3) The worst case of TD-Split and TD-Merge is making all RNs in a group as in TD-Aggr; therefore, the boundary condition for the three top-down schemes is the same, i.e., UE's PSE $=0.5$ and RN's PSE $=0.2$ as displayed in Fig. 15, in which the UEs awake 5 out of 10 subframes for receiving data from the RN, and the RNs awake 8 out of 10 subframes for receiving data from the DeNB (3 subframes) and transmitting data to the UEs (5 subframes). The same boundary condition applies to the bottom-up schemes.

(4) The mechanism of splitting (TD-Split) results in similar RN's and UE's PSE as the mechanism of merging (TD-Merge) in the top-down approach, but BU-Split outperforms BU-Merge as the input load reaches 19.2 Mbps as displayed in Fig. 17. The main reason is the cycle length in BU-Merge needs to be a power of 2 which usually leads to the reduction of the cycle length and therefore it's more likely to fail in schedulability check.

In order to compare the top-down approach with the bottom-up approach under equal load, Figs. 19, 20, and 21 display PSE of counterpart schemes in both approaches. It

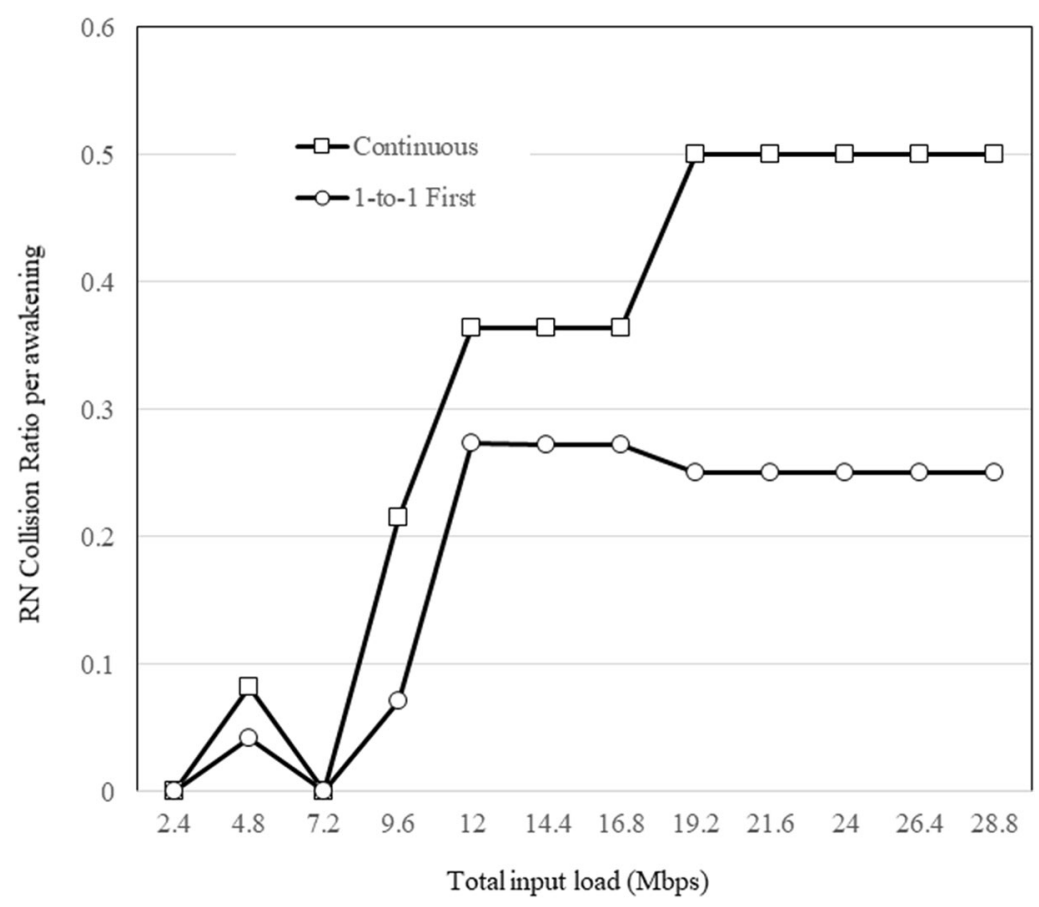

Fig. 11 RN collision of TD-Aggr-TD-Aggr RN collision simulation results with backhaul CQI = 15 and access CQI = 13 


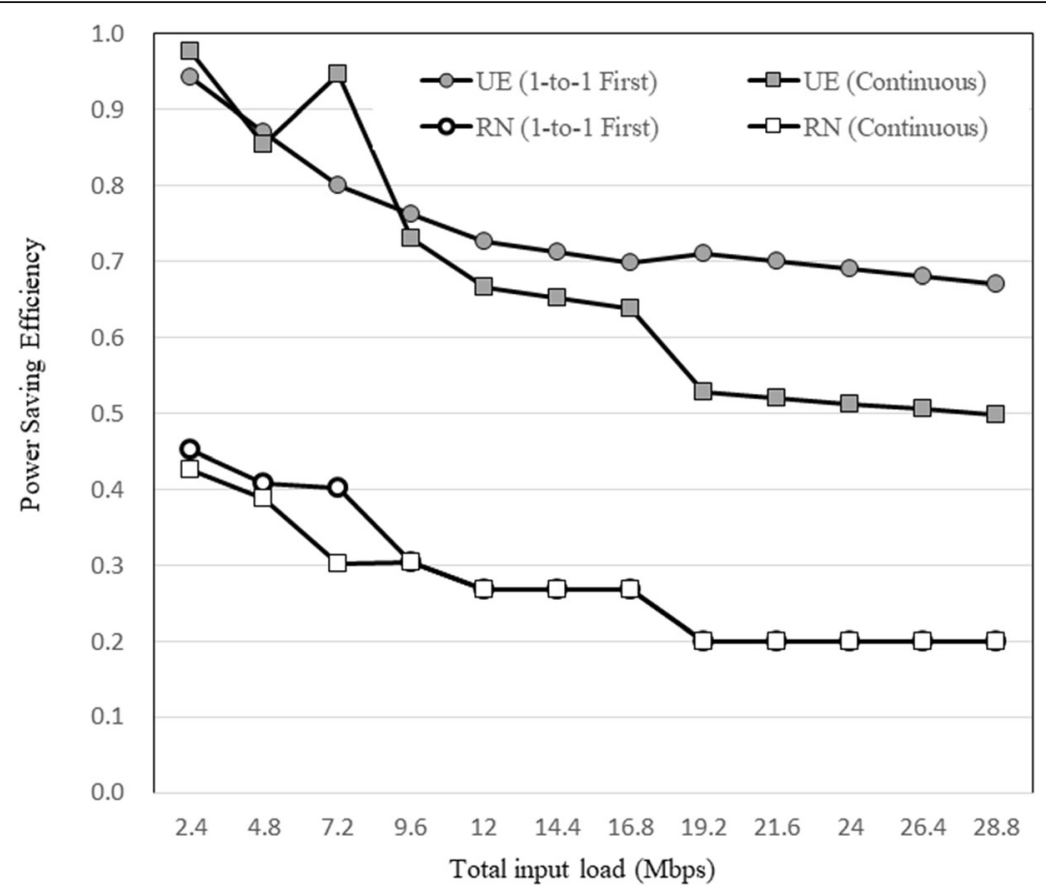

Fig. 12 PSE of TD-Aggr-TD-Aggr PSE simulation results with backhaul CQI = 15 and access CQI = 13

is shown that BU-Aggr and BU-Split outperform their counterpart top-down schemes. However, BU-Merge does not outperform TD-Merge as the input load is higher than 19.2 Mbps. To summarize, BU-Split is the best scheme under equal load.

\subsection{Results under hot spot}

Figures 22 and 23 display the results of PSE for the proposed schemes under hot spot. TD-Merge is the best scheme in terms of RN's PSE among top-down schemes, showing the benefit of allowing different

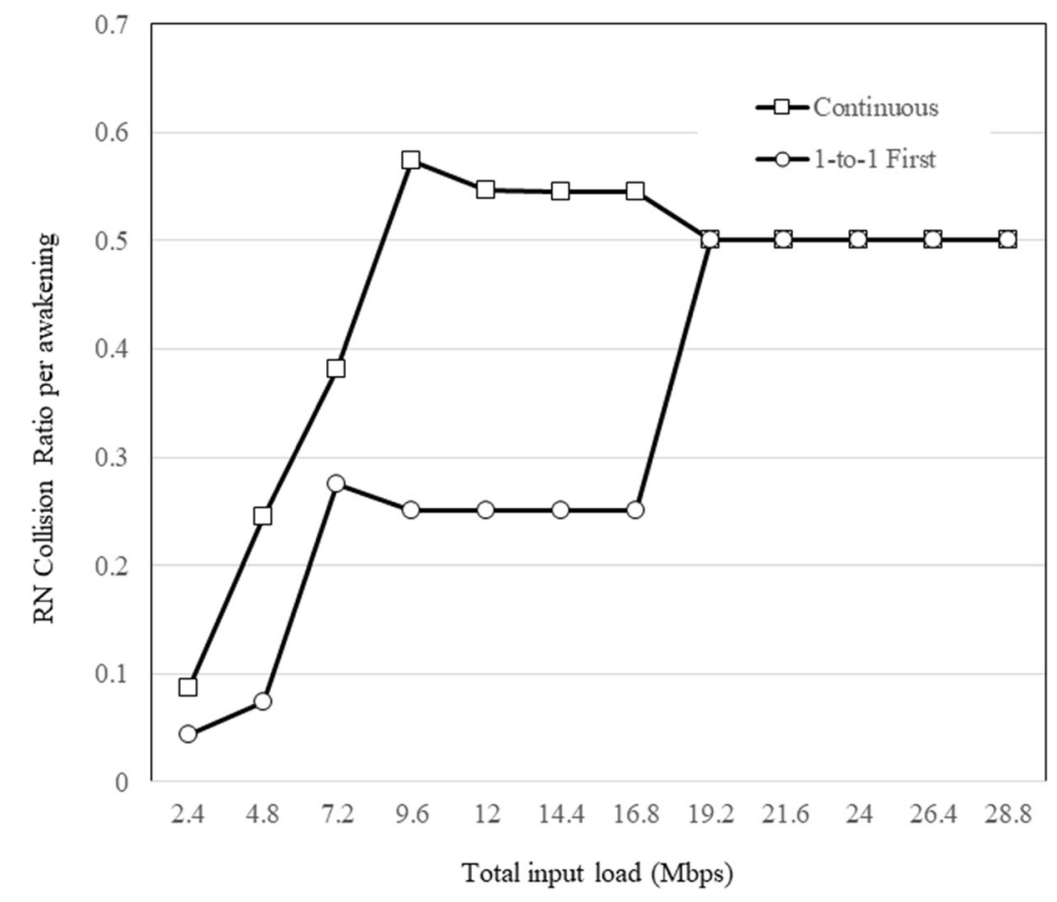

Fig. 13 RN collision of TD-Aggr-TD-Aggr RN collision simulation results with backhaul CQI = 10 and access CQI = 9 
Fig. 14 PSE of TD-Aggr-TD-Aggr PSE simulation results with backhaul CQI $=10$ and access CQI = 9

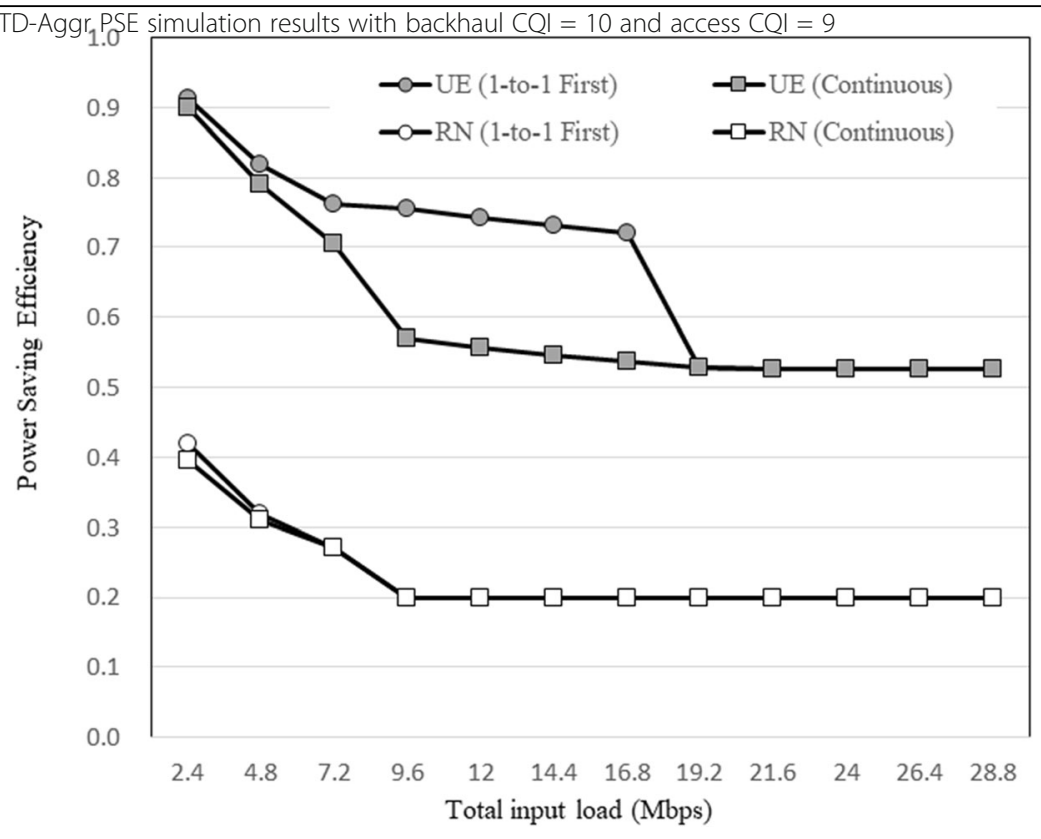

cycle length for different RN. On the other hand, as shown in Fig. 23, BU-Split is the best scheme among the bottom-up schemes. PSE of the top-down schemes and their counterpart bottom-up schemes is compared in Figs. 24, 25, and 26. These figures show that the bottom-up schemes do not always outperform their counterpart top-down schemes, since under hot spot the two RNs with heavier load place a bottleneck in schedulability check as the input load increases in the bottom-up approach. To summarize, BU-Split is still the best scheme in terms of PSE for most cases under hot spot.

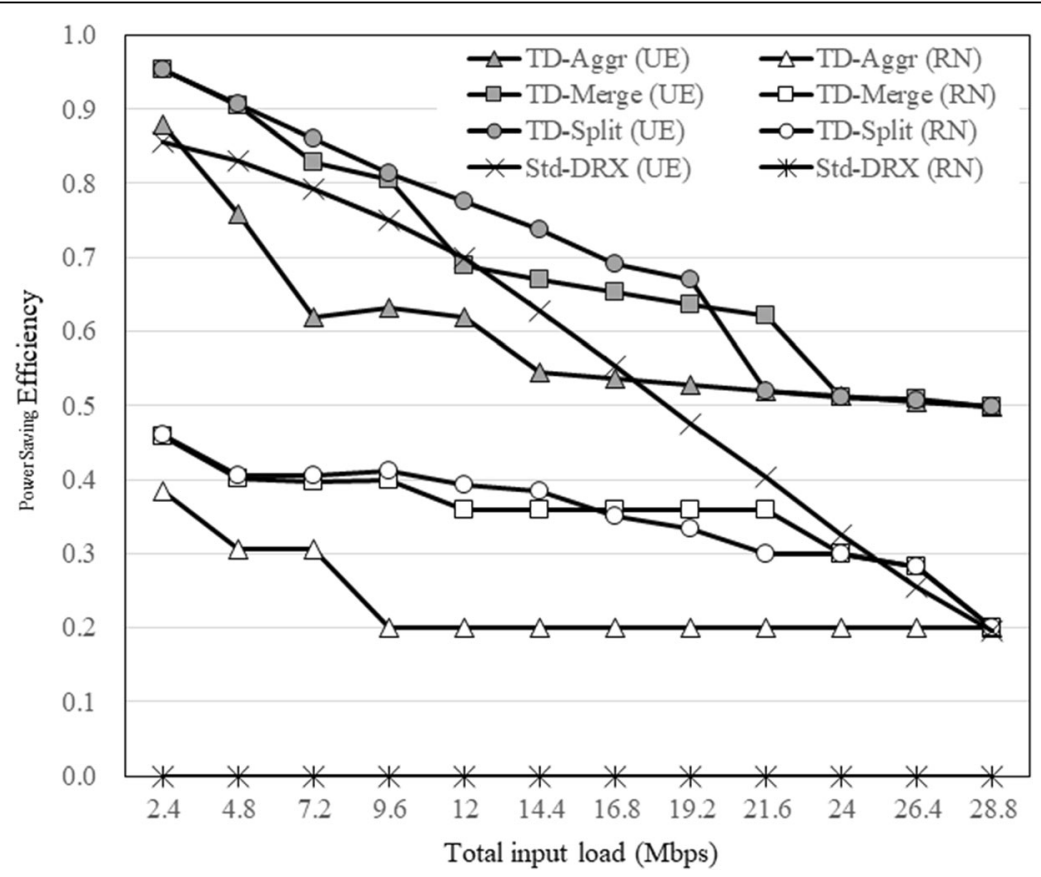

Fig. 15 PSE of top-down schemes-top-down PSE simulation results with equal load 


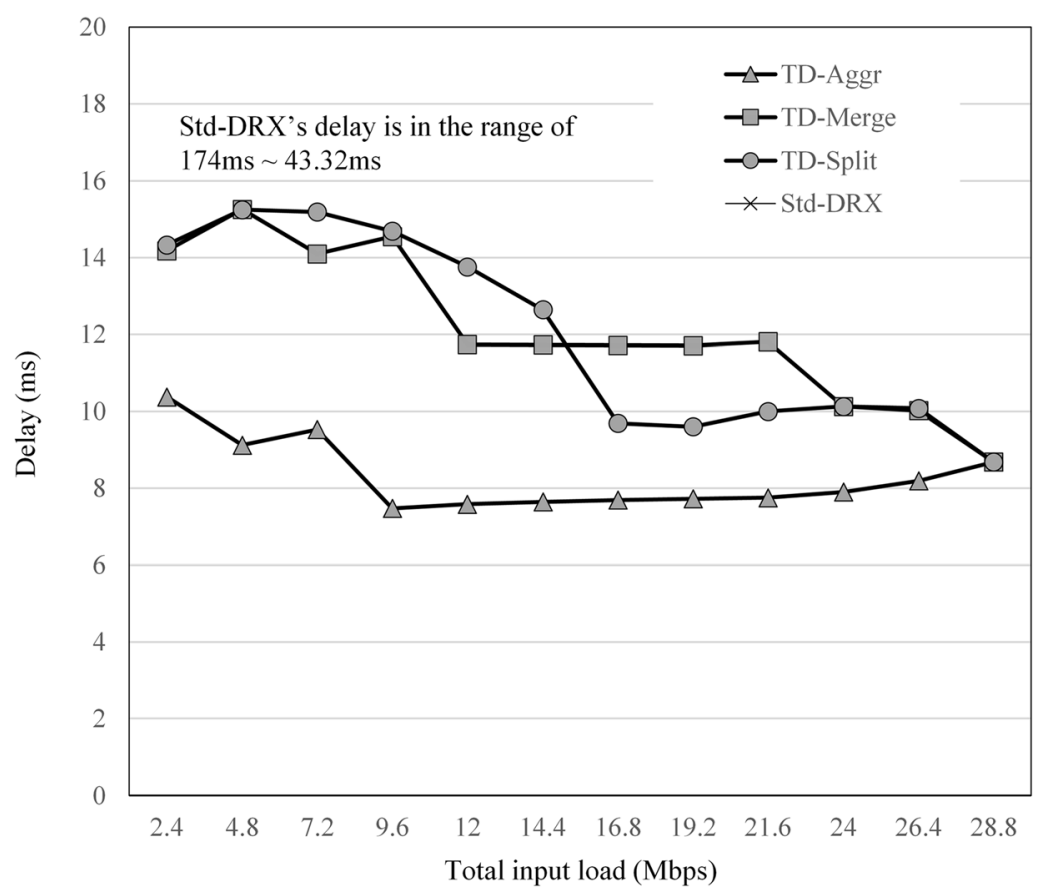

Fig. 16 Delay of top-down schemes - top-down delay simulation results with equal load

\subsection{Discussion on complexity and performance}

Since the six proposed schemes are based on the three previously proposed LBPS schemes of LBPS-Aggr, LBPSMerge, and LBPS-Split, understanding the characteristics of the LBPS schemes is the key for complexity analysis. LBPS-Aggr treats scheduling objects (RNs or UEs) as a single entity in sleep scheduling, by which the final sleep schedule can be determined in just one iteration of computation. On the other hand, LBPS-Split and LBPS-Merge require multiple iterations by splitting or merging objects to determine the final schedule. Starting from a single group containing all objects, LBPS-Split gradually reduces

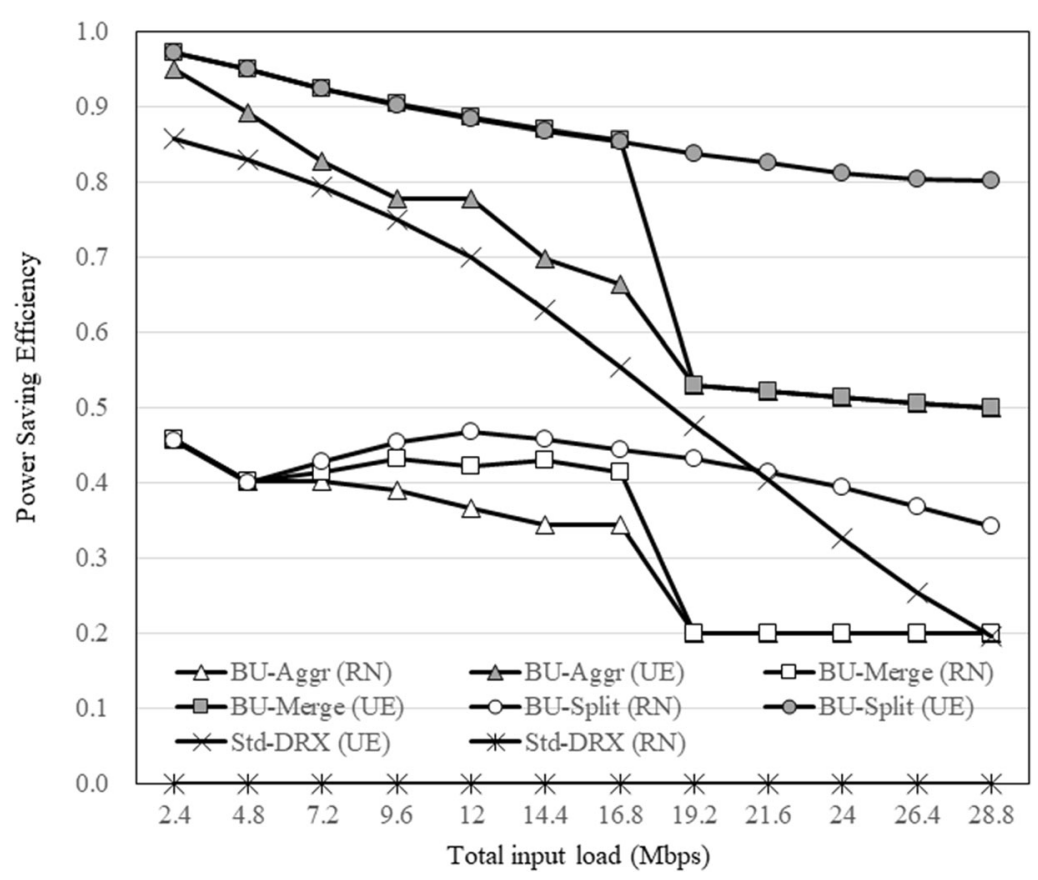

Fig. 17 PSE of bottom-up schemes-bottom-up PSE Simulation results with equal load 


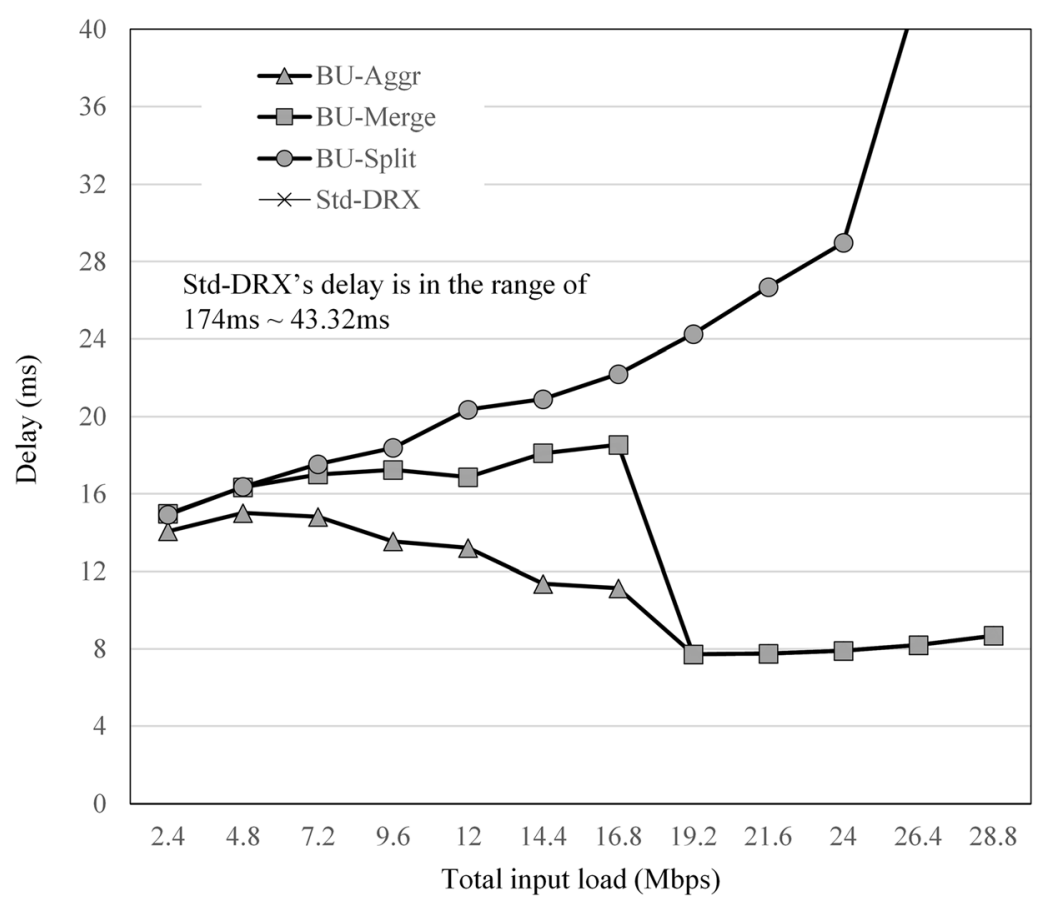

Fig. 18 Delay of bottom-up schemes-bottom-up delay simulation results with equal load

the group size until a feasible schedule is found. The maximum number of iterations in LBPS-Split is equal to the number of objects as the group size in the final schedule is reduced to one. In the opposite direction of LBPS-Split, LBPS-Merge starts from group size one and merges groups until a feasible schedule is found. In each iteration of LBPS-Merge, the number of groups is reduced to a half of the previous one. Therefore, the maximum number of iterations is $\log _{2}(m)$, where $m$ is the total number of scheduling objects.

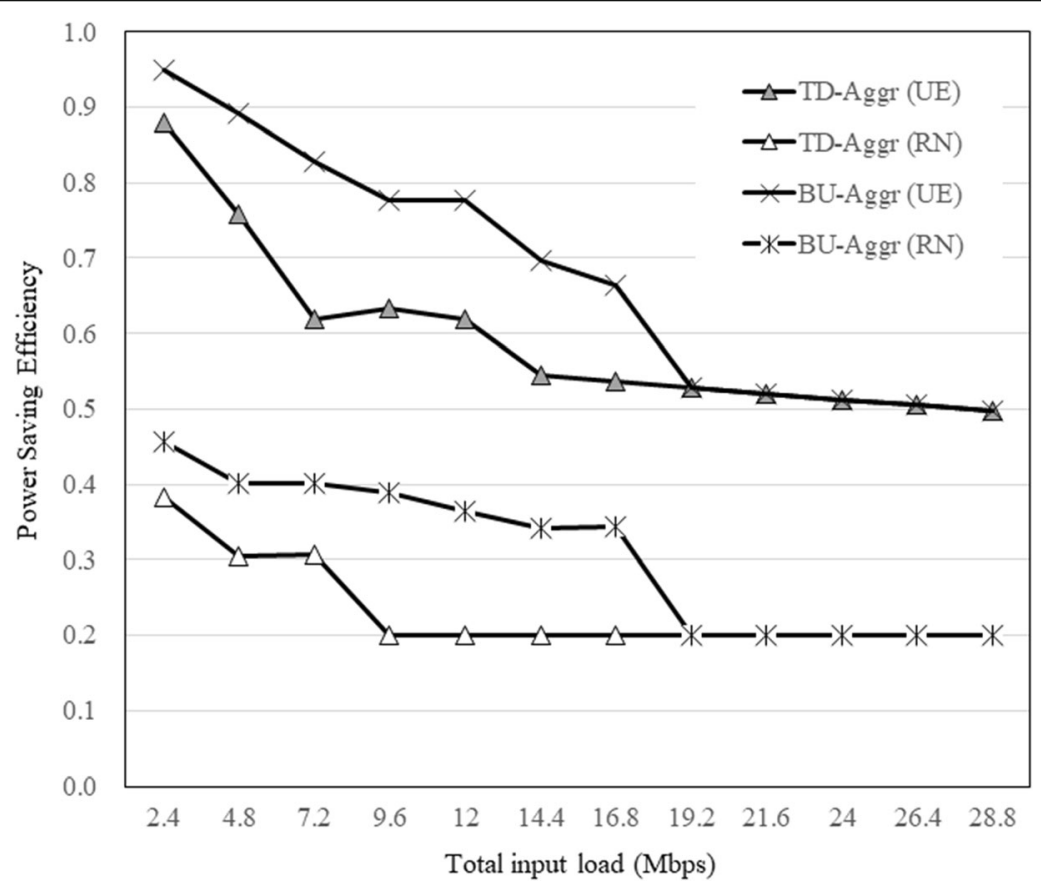

Fig. 19 TD-Aggr vs. BU-Aggr-TD-Aggr and BU-Aggr comparison with equal load 


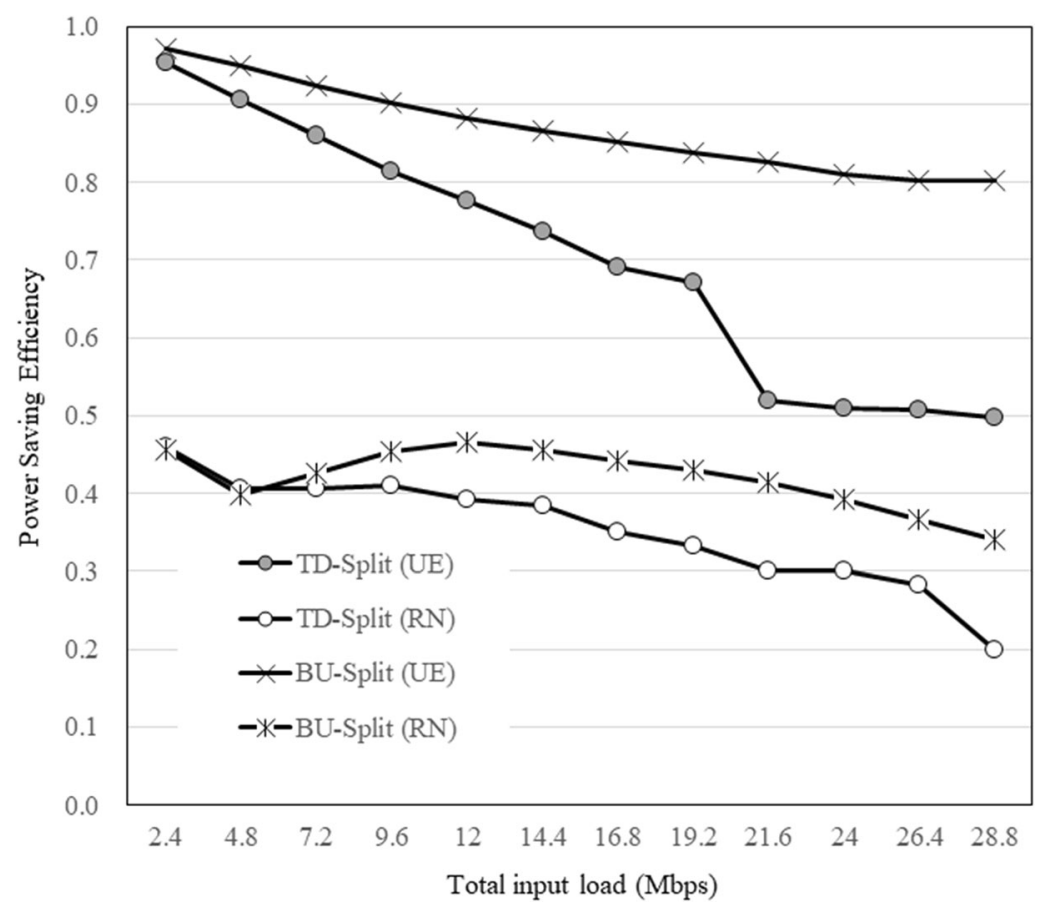

Fig. 20 TD-Split vs. BU-Split-TD-Split and BU-Split comparison with equal load

The first step in the top-down schemes of TD-Aggr, TD-Merge, and TD-Split is similar to their corresponding predecessor. The second step in the three top-down schemes is similar and requires only one iteration per $\mathrm{RN}$ to determine the final schedule on each access link. Therefore, assuming the number of RNs under the DeNB is $m$, the maximum number of iterations in total for each of the top-down schemes is $1+m$ for TD-Aggr, $\log _{2}(m)+m$ for TD-Merge, and $m+m$ for TD-Split.

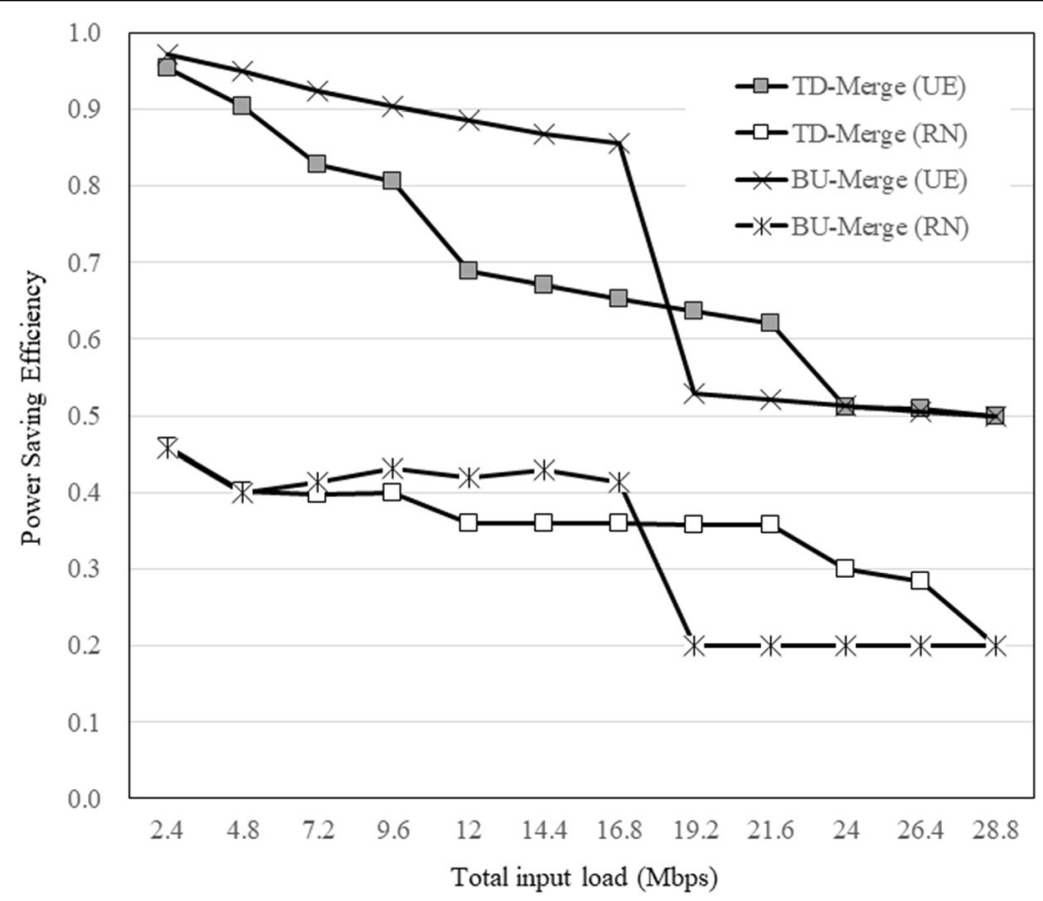

Fig. 21 TD-Merge vs. BU-Merge-TD-Merge and BU-Merge comparison with equal load 


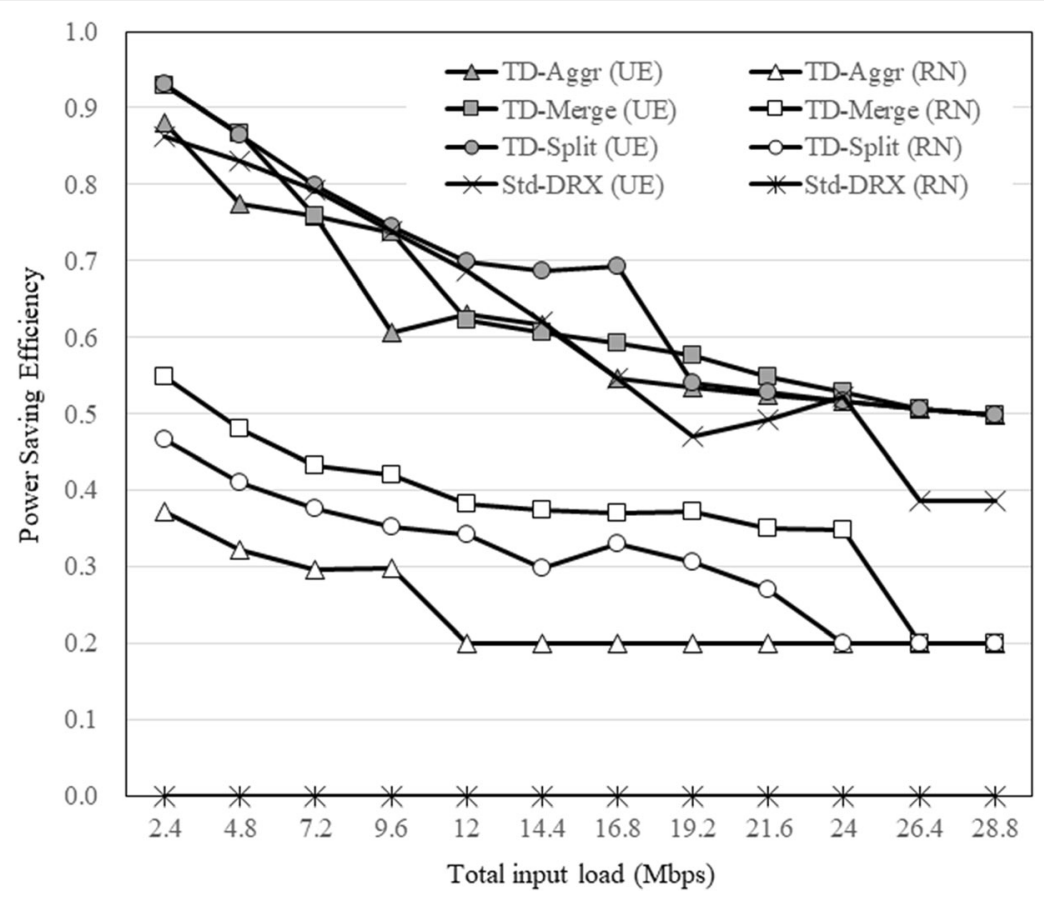

Fig. 22 PSE of top-down schemes-Top-down PSE simulation results with hot spot

The first step in the bottom-up schemes of BU-Aggr, BU-Merge, and BU-Split is similar to their corresponding predecessor. Note that the first step in the bottom-up schemes is applied to each access link. The second step in the three bottom-up schemes is similar and requires only one iteration. Therefore, assuming the number of UEs under each RN is $n$ (and the number of RNs is $m$ ), the maximum number of iterations in total for each of the bottom-up schemes is $m+1$ for BU-Aggr, $m \log _{2}(n)+1$ for BU-Merge, and $m n+1$ for BU-Split.

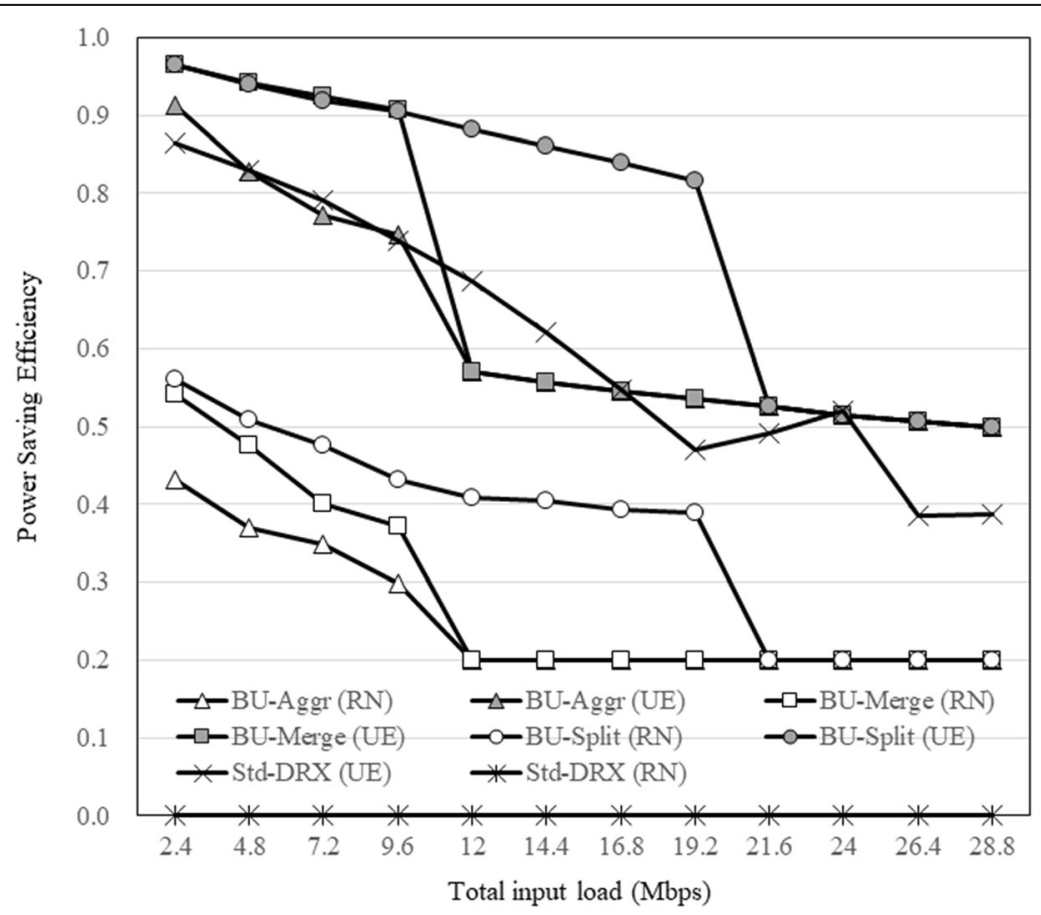

Fig. 23 PSE of bottom-up schemes-Bottom-up PSE simulation results with hot spot 


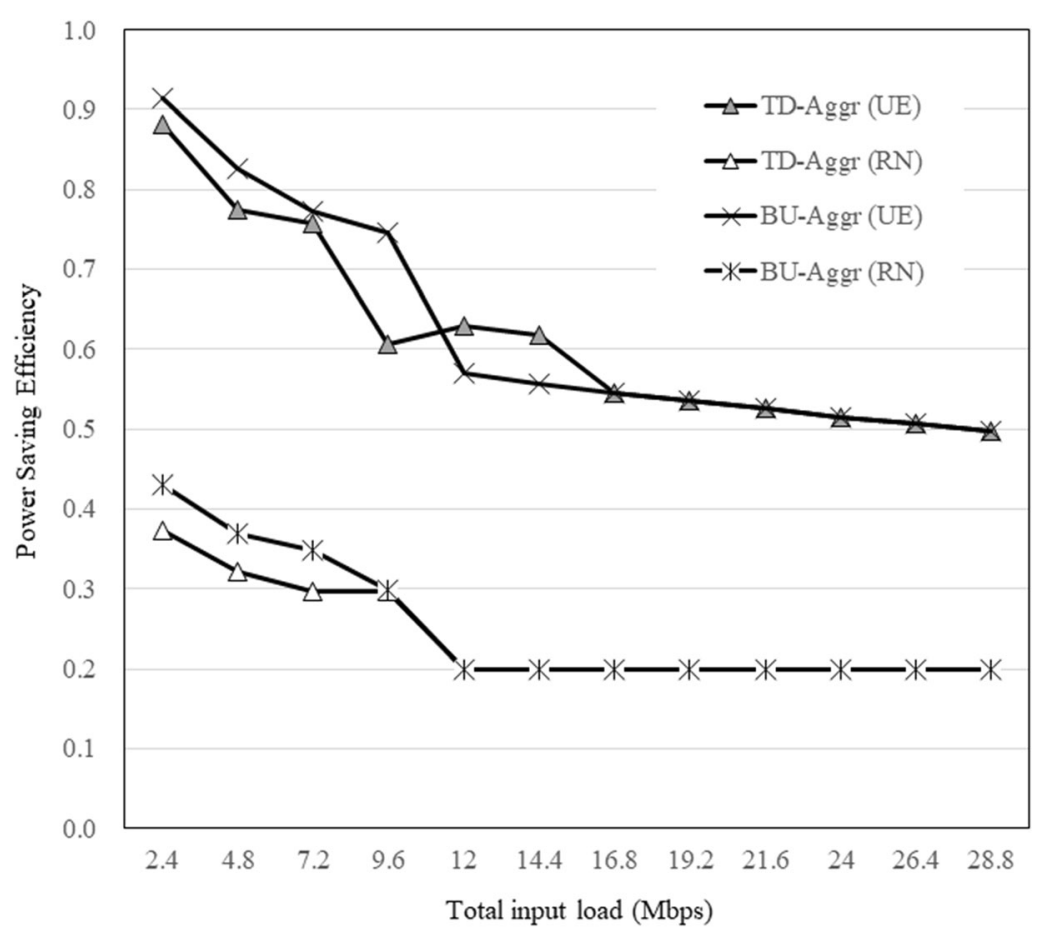

Fig. 24 TD-Aggr vs. BU-Aggr-TD-Aggr and BU-Aggr comparison with hot spot

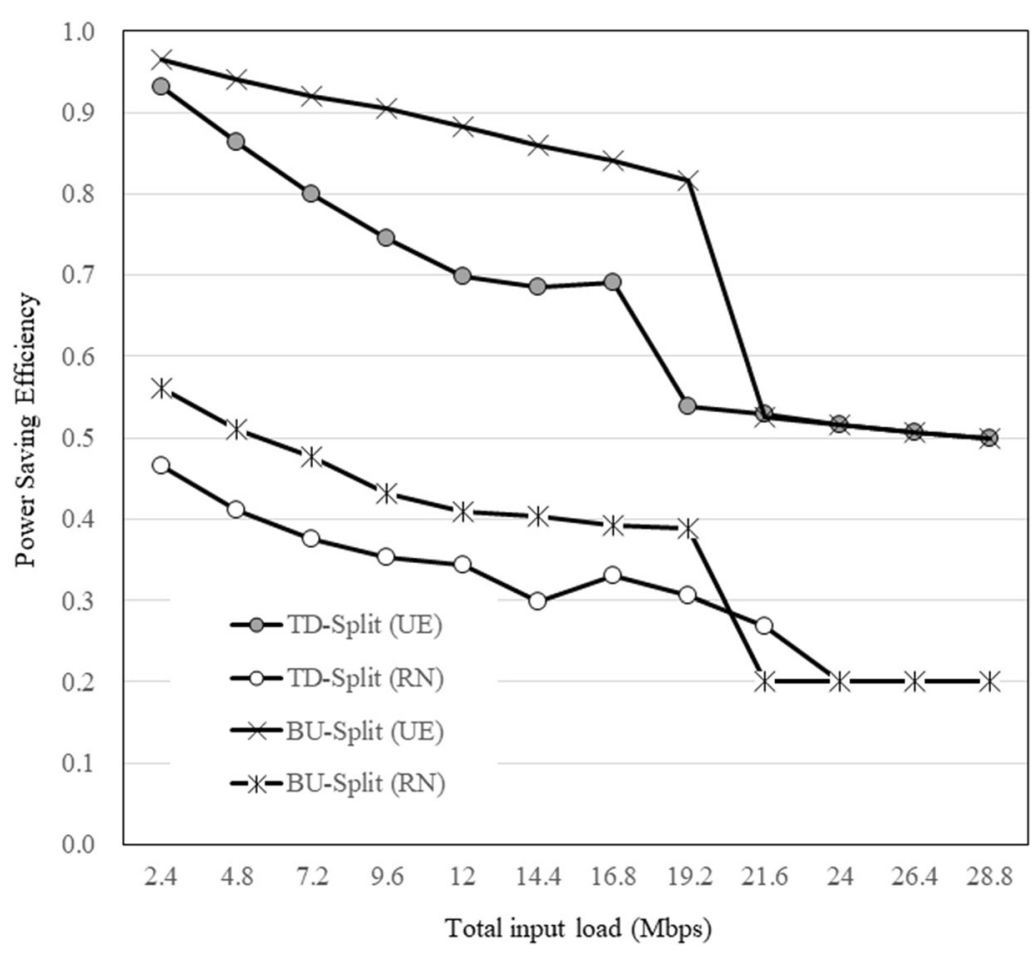

Fig. 25 TD-Split vs. BU-Split-TD-Split and BU-Split comparison with hot spot 


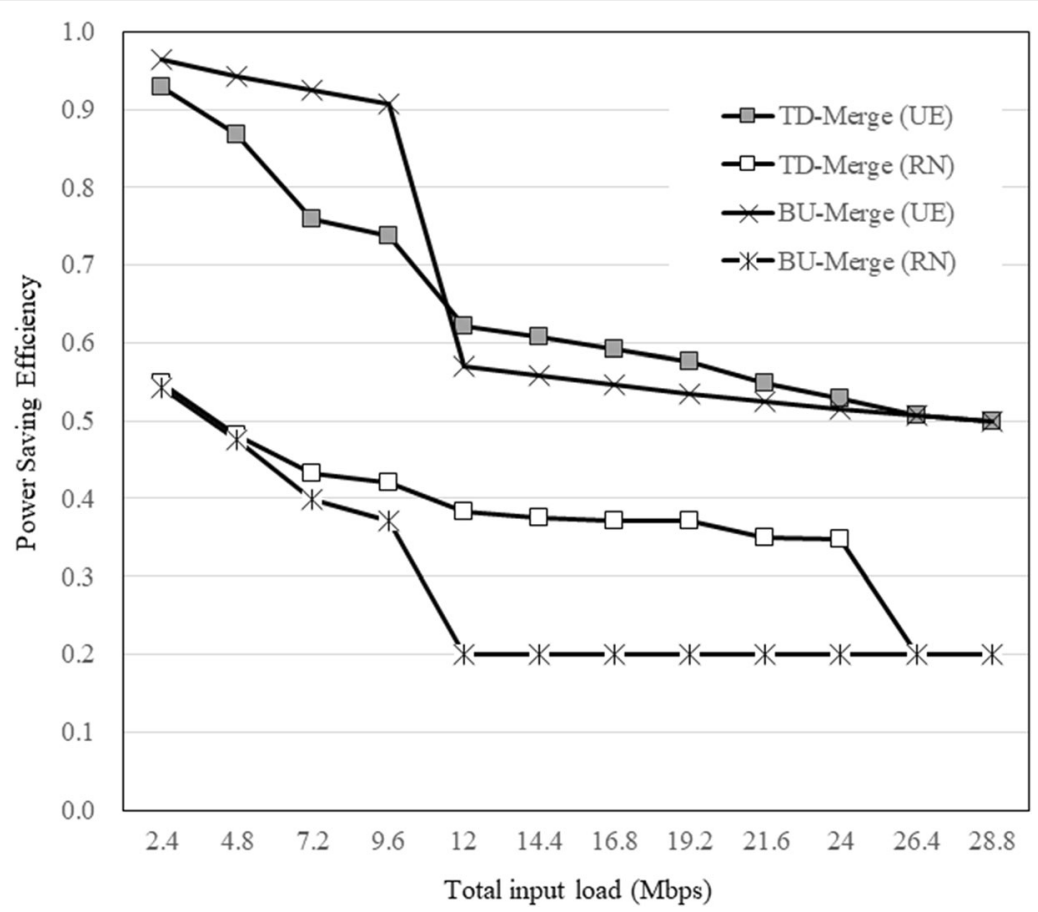

Fig. 26 TD-Merge vs. BU-Merge-TD-Merge and BU-Merge comparison with hot spot

A summary of complexity analysis for the proposed schemes is displayed in Table 5. In addition, performance data from the simulation study is also included in the table, in which TD-Aggr is selected as the baseline for performance normalization, and only the maximum figure for the other schemes is displayed. The complexity of the proposed schemes is in the following order: TD-Aggr $=\mathrm{BU}$ Aggr < TD-Merge < TD-Split < BU-Merge < BU-Split. Two concluding remarks can be made from Table 5 . Firstly, BU-Split achieves the highest PSE among the proposed schemes, but its computational cost and delay performance are much higher than the others. Secondly, BU-Aggr and TD-Merge are good candidates to provide a better balance between the computational cost and the performance.

\section{Conclusion}

This paper is focused on the energy-saving issue for the LTE-A network with relay nodes (RN) in time division duplex (TDD) is addressed. By extending the previous work of load-based power saving, we propose integrated sleep scheduling schemes covering RNs and UEs under the top-down and bottom-up strategies. Three top-down schemes, namely TD-Aggr, TD-Split, and TD-Merge, and three bottom-up schemes, namely, BU-Aggr, BUSplit, and BU-Merge, are proposed. In order for the proposed schemes to operate in TDD, the idea of virtual time is adopted. Given the TDD configuration, calculation of virtual subframe capacity as well as the mapping schemes from the virtual time to the actual time are presented. Two mapping schemes from the virtual time to

Table 5 Complexity analysis and performance comparison

\begin{tabular}{|c|c|c|c|c|c|c|}
\hline & \multicolumn{3}{|c|}{ Complexity (\# of iterations in the worst case) } & \multicolumn{3}{|c|}{ Performance (under equal load) } \\
\hline & First step & Second step per RN & Total & RN'S PSE & UE'S PSE & Delay \\
\hline TD-Aggr & 1 & 1 & $1+m$ & 1 & 1 & 1 \\
\hline TD-Merge & $\log _{2}(m)$ & 1 & $\log _{2}(m)+m$ & 2.0 & 1.34 & 1.95 \\
\hline \multirow[t]{2}{*}{ TD-Split } & $m$ & 1 & $m+m$ & 2.06 & 1.39 & 1.97 \\
\hline & First step per RN & Second step & & & & \\
\hline BU-Aggr & 1 & 1 & $m+1$ & 1.92 & 1.34 & 1.81 \\
\hline BU-Merge & $\log _{2}(n)$ & 1 & $m \log _{2}(n)+1$ & 2.16 & 1.59 & 2.41 \\
\hline BU-Split & $n$ & 1 & $m n+1$ & 2.33 & 1.61 & 5.12 \\
\hline
\end{tabular}

Note: $m$ is the number of RNs under the DeNB, and $n$ is the number of UEs under each RN 
the actual time, namely, 1-to-1 first mapping and continuous mapping, are proposed for the two-hop downlink transmission. Simulation study shows that 1-to-1 first mapping results in fewer RN collisions and achieves higher power-saving efficiency. It also shows that the six proposed schemes outperform the standard DRX scheme in terms of power-saving as well as the average delay. Moreover, TD-Split and BU-Split outperforms the other schemes in the same strategy in terms of powersaving but at the cost of larger average delay. On the other hand, bottom-up schemes outperform their counterpart top-down schemes. Moreover, the tradeoff between the computational cost and the performance for the proposed schemes is discussed in the paper. We conclude that BU-Split is the best scheme in power saving but with much higher computational cost. TDMerge and BU-Aggr can provide a better balance between the processing overhead and the performance.

\section{Abbreviations \\ 3GPP: 3rd Generation Partnership Project; CQI: Channel Quality Indicator; DeNB: Donor eNodeB; DL: Downlink; DRX: Discontinuous Reception; FDD: Frequency division duplex; HARQ: Hybrid automatic repeat request; LBPS: Load-based power saving; LTE: Long-Term Evolution; LTE-A: Long-Term Evolution-Advanced; MBSFN: Multimedia Broadcast Single-Frequency Network; PSE: Power saving efficiency; RB: Resource block; RN: Relay node; TDD: Time division duplex; UE: User Equipment; UL: Uplink; VSC: Virtual subframe capacity}

\section{Acknowledgements}

Preliminary version of this paper has been published in the proceedings of 19th International Conference on Mobile Communication Networks and Security (ICMCNS), March 27-28, 2017, Tokyo, Japan.

\section{Authors' contributions}

CCY, YTM. and JYC contributed to the main idea and drafted the manuscript, algorithm design, and performance analysis. CMY contributed to the simulations. All authors read and approved the final manuscript.

\section{Funding}

This work was supported in part by the Ministry of Science and Technology, Taiwan, R.O.C., under grant no. MOST 105-2221-E-260-013.

\section{Availability of data and materials}

Not applicable.

\section{Competing interests}

The authors declare that they have no competing interests.

\section{Author details}

'Department of Computer Science and Information Engineering, National Chi Nan University, Puli, Nantou County, Taiwan, Republic of China. ${ }^{2}$ Department of Sport Management, National Taiwan University of Sport, Taichung City, Taiwan, Republic of China. ${ }^{3}$ Department of Information Networking Technology, Hsiuping University of Science and Technology, Taichung City, Taiwan, Republic of China.

Received: 24 January 2019 Accepted: 13 August 2019

Published online: 06 September 2019

\section{References}

1. 3GPP TS 36.300, Evolved Universal Terrestrial Radio Access (E-UTRA) and Evolved Universal Terrestrial Radio Access Network (E-UTRAN). Rel. 8, v8.5.0 (2008).
2. 3GPP TS 36.300, Evolved Universal Terrestrial Radio Access (E-UTRA) and Evolved Universal Terrestrial Radio Access Network (E-UTRAN). Rel. 10, v10.3.0, (2011).

3. C.-C. Yang, Y.-T. Mai, J.-Y. Chen, Y.-S. Shen, Y.-C. Kuo, LBPS: Loadbased Power Saving in the IEEE 802.16e Network. Comput. Electr. Eng. 38(4), 891-905 (2012)

4. C.-C. Yang, J.-Y. Chen, Y.-T. Mai, C.-H. Liang, Adaptive load-based and channel-aware power saving for non-real-time traffic in LTE. EURASIP J. Wirel. Commun. Netw. 2015(1) (2015)

5. C.-C. Yang, J.-Y. Chen, Y.-T. Mai, Z.-Y. Pan, Integrated load-based power saving for real-time and non-real-time Traffic in LTE-TDD. Int. J. Commun. Syst. 30(15), 1-13 (2017)

6. Z. Ma, W. Xiang, H. Long, W. Wang, in Proceedings, IEEE International Conference on Communications (ICC). Proportional fair resource partition for LTE-advanced networks with type I relay nodes (2011), pp. 1-5. https:// ieeexplore.ieee.org/abstract/document/5963280

7. Z.Y. Zhao, J. Wang, S. Redana, B. Raaf, in Proceedings, IEEE Vehicular Technology Conference (VTC Fall). Downlink resource allocation for LTEadvanced networks with type1 relay nodes (2012), pp. 1-5. https:// ieeexplore.ieee.org/document/6399214

8. X.-L. Wu, W.-J. Zhao, W. Wu, in Proceedings, International Workshop on High Mobility Wireless Communications (HMWC). Throughput and fairnessbalanced resource allocation algorithm in TD-LTE-Advanced relay-enhanced network (2013), pp. 82-86. https://ieeexplore.ieee.org/document/6710316

9. L. Yang, A. Harada, in Proceedings, IEEE Wireless Communications and Networking Conference (WCNC). A spectrum allocation scheme for adjacent channel interference mitigation in relay backhaul link in coexisting FDD and TDD systems (2013), pp. 516-521. https://ieeexplore.ieee.org/ document/6554617

10. Y. Yuda, A. Iwata, D. Imamura, in Proceedings, IEEE International Conference on Communications (ICC). Interference mitigation using coordinated backhaul timing allocation for LTE-advanced relay systems (2011), pp. 1-5. https://ieeexplore.ieee.org/document/5963129

11. W. Hong, J. Han, H. Wang, in Proceedings, IEEE Vehicular Technology Conference (VTC Fall). Full uplink performance evaluation of FDD/TDD LTEadvanced networks with type-1 relays (2011), pp. 1-5. https://ieeexplore. ieee.org/abstract/document/6092869

12. L. Jiang, M. Lei, J. Du, in Proc. IEEE 77th Vehicular Technology Conference (VTC Spring). Cross-subframe co-channel interference mitigation scheme for LTEadvanced dynamic TDD dystem (2013), pp. 1-5. https:/ieeexplore.ieee.org/ abstract/document/6692497

13. M. Ding, D.L. Pérez, A.V. Vasilakos, W. Chen, in Proceedings, IEEE International Conference on Communications Workshops (ICC). Dynamic TDD transmissions in homogeneous small cell networks (2014), pp. 616-621. https://ieeexplore. ieee.org/document/6881267

14. H. Liu, Y. Jiao, Y. Gao, L. Sang, D. Yang, in Proceedings, 22nd International Conference on Telecommunications (ICT). Performance evaluation of flexible duplex implement based on radio frame selection in LTE heterogeneous network (2015), pp. 308-312. https://ieeexplore.ieee.org/document/7124702

15. M. Malmirchegini, R. Yenamandra, K.R. Chaudhuri, J.E.V. Bautista, in Proceedings, IEEE 81st Vehicular Technology Conference (VTC Spring). Distributed and adaptive optimization of LTE-TDD configuration based on UE traffic type (2015), pp. 1-6. https://ieeexplore.ieee.org/document/7145782

16. Y. Lan, A. Harada, in Proc. IEEE Wireless Communications and Networking Conference (WCNC). A spectrum allocation scheme for adjacent channel interference mitigation in relay backhaul link in coexisting FDD and TDD systems (2013), pp. 516-521. https://www.semanticscholar.org/paper/Aspectrum-allocation-scheme-for-adjacent-channel-Lan-Harada/f1 10b09bc54 0c04747b42f677c8d80af17506308

17. W. Hong, J. Han, H. Wang, in Proc. IEEE Vehicular Technology Conference (VTC Fall). Full uplink performance evaluation of FDD/TDD LTE-advanced networks with type-1 relays (2011), pp. 1-5. https://ieeexplore.ieee.org/ document/6092869

\section{Publisher's Note}

Springer Nature remains neutral with regard to jurisdictional claims in published maps and institutional affiliations. 